TRANSACTIONS OF THE

AMERICAN MATHEMATICAL SOCIETY

Volume 353, Number 3, Pages 1055-1087

S 0002-9947(00)02605-2

Article electronically published on November 8, 2000

\title{
A SHARP BOUND FOR THE RATIO OF THE FIRST TWO DIRICHLET EIGENVALUES OF A DOMAIN IN A HEMISPHERE OF $\mathbb{S}^{n}$
}

\author{
MARK S. ASHBAUGH AND RAFAEL D. BENGURIA
}

\begin{abstract}
For a domain $\Omega$ contained in a hemisphere of the $n$-dimensional sphere $\mathbb{S}^{n}$ we prove the optimal result $\lambda_{2} / \lambda_{1}(\Omega) \leq \lambda_{2} / \lambda_{1}\left(\Omega^{\star}\right)$ for the ratio of its first two Dirichlet eigenvalues where $\Omega^{\star}$, the symmetric rearrangement of $\Omega$ in $\mathbb{S}^{n}$, is a geodesic ball in $\mathbb{S}^{n}$ having the same $n$-volume as $\Omega$. We also show that $\lambda_{2} / \lambda_{1}$ for geodesic balls of geodesic radius $\theta_{1}$ less than or equal to $\pi / 2$ is an increasing function of $\theta_{1}$ which runs between the value $\left(j_{n / 2,1} / j_{n / 2-1,1}\right)^{2}$ for $\theta_{1}=0$ (this is the Euclidean value) and $2(n+1) / n$ for $\theta_{1}=\pi / 2$. Here $j_{\nu, k}$ denotes the $k$ th positive zero of the Bessel function $J_{\nu}(t)$. This result generalizes the Payne-Pólya-Weinberger conjecture, which applies to bounded domains in Euclidean space and which we had proved earlier. Our method makes use of symmetric rearrangement of functions and various technical properties of special functions. We also prove that among all domains contained in a hemisphere of $\mathbb{S}^{n}$ and having a fixed value of $\lambda_{1}$ the one with the maximal value of $\lambda_{2}$ is the geodesic ball of the appropriate radius. This is a stronger, but slightly less accessible, isoperimetric result than that for $\lambda_{2} / \lambda_{1}$. Various other results for $\lambda_{1}$ and $\lambda_{2}$ of geodesic balls in $\mathbb{S}^{n}$ are proved in the course of our work.
\end{abstract}

\section{INTRODUCTION}

With our earlier proof [5, 6], 7] of the Payne-Pólya-Weinberger (PPW) conjecture [42], [43] the bound

$$
\lambda_{2} / \lambda_{1}(\Omega) \leq \lambda_{2} / \lambda_{1}\left(\Omega^{\star}\right)=j_{n / 2,1}^{2} / j_{n / 2-1,1}^{2}
$$

was established for the ratio of the first two eigenvalues of the Laplacian $-\Delta$ on a bounded domain $\Omega \subset \mathbb{R}^{n}$ with Dirichlet boundary conditions imposed on $\partial \Omega$. Here $\Omega^{\star}$ represents the $n$-dimensional ball having the same volume as $\Omega$ (but, in fact, by scaling any ball will do) and $j_{\nu, k}$ represents the $k$ th positive zero of the Bessel function $J_{\nu}(t)$ [1]. Equality obtains in (1.1) if and only if $\Omega$ is a ball to begin with.

Received by the editors January 6, 1999 and, in revised form, June 9, 1999.

1991 Mathematics Subject Classification. Primary 58G25; Secondary 35P15, 49Rxx, 33C55.

Key words and phrases. Eigenvalues of the Laplacian, Dirichlet problem for domains on spheres, Payne-Pólya-Weinberger conjecture, Sperner's inequality, ratios of eigenvalues, isoperimetric inequalities for eigenvalues.

The first author was partially supported by National Science Foundation (USA) grants DMS9114162, INT-9123481, DMS-9500968, and DMS-9870156.

The second author was partially supported by FONDECYT (Chile) project number 196-0462, a Cátedra Presidencial en Ciencias (Chile), and a John Simon Guggenheim Memorial Foundation fellowship. 
In this paper we prove the analog of this result for domains in a hemisphere of $\mathbb{S}^{n}$ $\left(-\Delta\right.$ is now, of course, the Laplacian on $\left.\mathbb{S}^{n}\right)$.

It turns out to be better to view (1.1) as

$$
\lambda_{2}(\Omega) \leq \lambda_{2}\left(B_{\lambda_{1}}\right),
$$

where $B_{\lambda_{1}}$ represents the $n$-dimensional ball that has the value $\lambda_{1}(\Omega)$ as its first Dirichlet eigenvalue. This is, in fact, the way our proof proceeded $([5],[6]$, [8], see also [27]). Of course, the choice of ball here just involves choosing an appropriate radius and this choice is always unique since the first eigenvalue of a ball is a strictly decreasing function of its radius and goes from infinity to zero as the radius goes from zero to infinity. In words, (1.2) says that among all $n$-dimensional domains having the same first eigenvalue the $n$-dimensional ball has maximal second eigenvalue. One might compare this statement with the statement of the Faber-Krahn inequality [30, 37], 38]: among all $n$-dimensional domains having the same volume the $n$-dimensional ball has minimal first (Dirichlet) eigenvalue. Also of interest is the Szegö-Weinberger inequality [50], [52]: among all $n$-dimensional domains having the same volume the $n$-dimensional ball has maximal first nonzero Neumann eigenvalue. Both of these other inequalities are relevant here; the first because it figures in our proof and the second because in many ways the proof of this result is analogous to (though considerably simpler than) our proof of the Payne-PólyaWeinberger conjecture.

What we do in this paper is transfer the strategy outlined in the last paragraph over to bounded domains in $\mathbb{S}^{n}$ contained in hemispheres. It turns out that, properly interpreted, everything that was said above concerning domains in Euclidean space also holds in $\mathbb{S}^{n}$. Thus we prove that (1.2) holds if $\Omega$ is a domain in a hemisphere in $\mathbb{S}^{n}$ and $B_{\lambda_{1}}$ is the geodesic ball in $\mathbb{S}^{n}$ having $\lambda_{1}(\Omega)$ as its first eigenvalue. There is a Faber-Krahn result in $\mathbb{S}^{n}$ [49] (see also [31]), too, and $\lambda_{1}$ of a geodesic ball is still strictly monotone decreasing and goes to infinity at zero so a unique $B_{\lambda_{1}}$ which is a hemisphere or less will exist. For the Faber-Krahn and Szego"-Weinberger inequalities in $\mathbb{S}^{n}$ one need only read "volume" as "canonical $n$-dimensional volume in $\mathbb{S}^{n}$ " and "ball" as "geodesic ball". The generalization of the Szego"-Weinberger result to domains in hemispheres of $\mathbb{S}^{n}$ is a recent result of ours [9] (see also prior work of Chavel [22]). Our proof here parallels the one in [9] as well as our proof of the Payne-Pólya-Weinberger conjecture [5], 6], [7] (see especially our proof in [7]) for the Euclidean case.

The biggest difference between our work on $\lambda_{2} / \lambda_{1}$ for domains in $\mathbb{S}^{n}$ versus those in $\mathbb{R}^{n}$ revolves around the difference between (1.1) and (1.2). In $\mathbb{R}^{n}$ (1.1) and (1.2) are equivalent since $\lambda_{2} / \lambda_{1}$ is the same for any ball, whatever its size. This follows from the fact that in that case the eigenvalues scale with the radius, in particular, for a ball of radius $R$ in $\mathbb{R}^{n} \quad \lambda_{1}=j_{n / 2-1,1}^{2} / R^{2}, \lambda_{2}=j_{n / 2,1}^{2} / R^{2}$, and hence $\lambda_{2} / \lambda_{1}=j_{n / 2,1}^{2} / j_{n / 2-1,1}^{2}$ and is independent of $R$. When one passes to $\mathbb{S}^{n}$ this is no longer the case. If we let $\theta_{1}$ denote the radius of our geodesic ball, then the first and second eigenvalues of that ball, which we denote by $\lambda_{1}\left(\theta_{1}\right)$ and $\lambda_{2}\left(\theta_{1}\right)$, are in general more complicated functions of $\theta_{1}$. By domain monotonicity (see, for example, 14], 23], 28], [44) these are, of course, strictly decreasing functions but more precise knowledge of them (or combinations thereof) requires considerable effort. For example, for the case of $\mathbb{S}^{n}$, to pass from (1.2) back to the first part of (1.1) (i.e., $\lambda_{2} / \lambda_{1}(\Omega) \leq \lambda_{2} / \lambda_{1}\left(\Omega^{\star}\right)$, where $\Omega^{\star}$ is the symmetric rearrangement of $\Omega$ on $\mathbb{S}^{n}$ ) one needs to know that $\lambda_{2} / \lambda_{1}$ for geodesic balls is an increasing function 
of $\theta_{1}$. We do this below in Section 3. In general, most of what could almost be taken for granted for the case of balls in $\mathbb{R}^{n}$ expands to some problem about how $\lambda_{1}, \lambda_{2}$, or a combination of the two behaves as a function of $\theta_{1}$. Another instance of this is that while for a ball in $\mathbb{R}^{n}$ it is easy to see that $\lambda_{2}$ corresponds to an $\ell=1$ eigenfunction (i.e., an eigenfunction associated with an $\ell=1$ spherical harmonic) in $\mathbb{S}^{n}$ the analog of this must be proved for all $\theta_{1} \in(0, \pi / 2]$. The proof of this fact, while not difficult, is given in Section 3. In fact, in Section 3 (see Lemma 3.1) we prove the result for all $\theta_{1} \in(0, \pi)$ (note that $m$ replaces $\ell$ there).

For orientation we outline the elements of our proof here. Aside from item 4 (which we have just discussed) these elements were also present in our proof in the Euclidean case.

1. Rayleigh-Ritz inequality for estimating $\lambda_{2}$ :

$$
\lambda_{2}-\lambda_{1} \leq \frac{\int_{\Omega}|\nabla P|^{2} u_{1}^{2} d \mu}{\int_{\Omega} P^{2} u_{1}^{2} d \mu}
$$

if $\int_{\Omega} P u_{1}^{2} d \mu=0$ and $P \not \equiv 0$. Here $u_{1}$ denotes the normalized eigenfunction for $\lambda_{1}$. This inequality applies on manifolds as in Euclidean space; one only has to view $|\nabla P|$ as a norm with respect to the metric on the manifold (see, e.g., 23], pp. 50-51). Also $d \mu$ represents the intrinsic volume element for the manifold. The inequality given here follows from the usual Rayleigh-Ritz inequality for $\lambda_{2}$ by taking $P u_{1}$ as a trial function and integrating by parts appropriately.

2. A Brouwer fixed point theorem argument that allows us to insure that the condition $\int_{\Omega} P u_{1}^{2} d \mu=0$ is satisfied for $n$ specific choices of the function $P$. In our recent paper [9] we gave a version of this argument for $\mathbb{S}^{n}$ which also applies here. In Section 2 of this paper we give an improved version of this argument (using degree theory rather than the Brouwer fixed point theorem) which yields a slightly stronger result. The original argument of this type (to our knowledge) was given by Weinberger in 52. For future reference, we note that all such results will be referred to as "center of mass results".

3. Rearrangement results for functions and domains. These results are measure theoretic in nature and easily extend to problems on manifolds. There is also an easily effected preliminary rearrangement in $\mathbb{S}^{n}$ (see also 8], 9]).

4. Properties of the eigenvalues and eigenfunctions of geodesic balls in $\mathbb{S}^{n}$. These we establish in Section 3 below. The details depend on Legendre and associated Legendre functions though we manage to keep these functions (also expressible in terms of hypergeometric functions) in the background. In $\mathbb{R}^{n}$, of course, the special functions that occur are Bessel functions.

5. Monotonicity properties of certain special combinations of the eigenfunctions for $\lambda_{1}$ and $\lambda_{2}$ for geodesic balls contained in hemispheres. These functions are special to the problem of maximizing $\lambda_{2} / \lambda_{1}$ (or $\lambda_{2}$ subject to $\lambda_{1}=$ const.) and in a technical sense their properties proved here are the most difficult part of our overall proof. These properties are proved in Section 4 below.

6. Chiti's comparison argument. This is a specialized comparison result which establishes a crossing property of the symmetric-decreasing rearrangement of

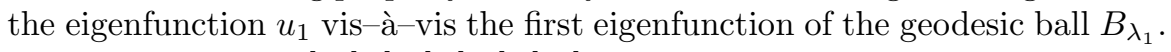
This result of Chiti [24], [25], [26], [27] for the Euclidean case is based upon a rearrangement technique for partial differential equations due to Talenti [51] 
which in turn is based on the classical isoperimetric inequality in $\mathbb{R}^{n}[21,[23$, [41]. All of these results generalize to $\mathbb{S}^{n}$. We give these arguments in detail in Section 5.

Our main results are summarized in the following theorems:

Theorem 1.1. Let $\Omega$ be contained in a hemisphere of $\mathbb{S}^{n}$ and let $B_{\lambda_{1}}$ denote the geodesic ball in $\mathbb{S}^{n}$ having the same value of $\lambda_{1}$ as $\Omega$ (i.e., $\lambda_{1}(\Omega)=\lambda_{1}\left(B_{\lambda_{1}}\right)$ ). Then

$$
\lambda_{2}(\Omega) \leq \lambda_{2}\left(B_{\lambda_{1}}\right)
$$

with equality if and only if $\Omega$ is itself a geodesic ball in $\mathbb{S}^{n}$.

Theorem 1.2. The first Dirichlet eigenvalue for a geodesic ball in $\mathbb{S}^{n}$ of geodesic radius $\theta_{1}, \lambda_{1}\left(\theta_{1}\right)$, is such that $\theta_{1}^{2} \lambda_{1}\left(\theta_{1}\right)$ is a decreasing function of $\theta_{1}$ for $0<\theta_{1} \leq \pi$.

Theorem 1.3. The quotient between the first two Dirichlet eigenvalues for a geodesic ball in $\mathbb{S}^{n}$, of geodesic radius $\theta_{1}, \lambda_{2} / \lambda_{1}$, is an increasing function of $\theta_{1}$ for $0<\theta_{1} \leq \pi / 2$.

Finally, we come to the PPW conjecture for domains in hemispheres of $\mathbb{S}^{n}$ :

Theorem 1.4. Let $\Omega$ be contained in a hemisphere of $\mathbb{S}^{n}$. Then

$$
\lambda_{2} / \lambda_{1}(\Omega) \leq \lambda_{2} / \lambda_{1}\left(\Omega^{\star}\right)
$$

with equality if and only if $\Omega$ is itself a geodesic ball in $\mathbb{S}^{n}$.

This theorem follows from Theorems 1.1 and 1.3 above. If $B_{\lambda_{1}}$ is a ball having the same $\lambda_{1}$ as $\Omega$, we have

$$
\lambda_{2} / \lambda_{1}(\Omega) \leq \lambda_{2} / \lambda_{1}\left(B_{\lambda_{1}}\right)=\lambda_{2}\left(\theta_{1}\right) / \lambda_{1}\left(\theta_{1}\right),
$$

where $\theta_{1}$ is the geodesic radius of $B_{\lambda_{1}}$. Theorem 1.4 now follows from Theorem 1.3 and Sperner's optimal Faber-Krahn-type result [49] for domains in $\mathbb{S}^{n}$, which implies that $\theta_{1}\left(\Omega^{\star}\right) \geq \theta_{1}\left(B_{\lambda_{1}}\right)$ (via domain monotonicity).

The results given in Theorems 1.1 1.4 were announced earlier in 8 and $[10$ (note, however, that Theorem 1.2 is not mentioned explicitly in 8, nor is Theorem 1.3 stated formally there). Moreover, 10 contains alternative proofs of some of the results proved here. These may be of independent interest, since they may point the way to generalizing the results presented here to other settings. We note, though, that the proofs contained herein are those by which we first proved the results stated above. In fact, there is a close parallel between the proofs given here and those of our "second" proof of the Euclidean PPW conjecture given in [7] and of our $\mathbb{S}^{n}$-version of the Szegö-Weinberger inequality given in [9]. One of our objectives in 8] was to bring these similarities to the fore.

Beyond this, and as alluded to in passing in the previous paragraph, one might ask to what extent our results above are optimal, and, further, whether or not they are amenable to generalization (for example, to other spaces, the most obvious being $\left.\mathbb{H}^{n}\right)$. In particular, one might ask if it might be possible to remove our restriction to domains contained in a hemisphere of $\mathbb{S}^{n}$. It will be apparent to anyone who studies our proofs that the success of our approach is very much dependent on this condition. Certainly it should take little convincing to see that geometrically things become quite different "beyond the hemisphere" : for example, up to the hemisphere both the volume and surface area of a geodesic ball are increasing functions, but beyond the hemisphere the volume continues to grow while the surface area actually shrinks. On the other hand, we do not have any counterexamples to the conjecture 
that our theorems above (aside from Theorem[1.2, which is already established for all geodesic balls, and not just those contained in a hemisphere) continue to hold in the absence of the hemisphere condition. In fact, we have been able to establish that Theorem 1.3.3 does hold for all $0<\theta_{1}<\pi$ for $n=2$ and 3. We might also note that it is common to encounter some sort of impediment at the hemisphere when dealing with the eigenvalues (specifically, Dirichlet or Neumann) of the Laplacian for domains in $\mathbb{S}^{n}$. For example, one might note that while for domains in Euclidean space and for domains strictly contained in a hemisphere of $\mathbb{S}^{n}$ the first nonzero Neumann eigenvalue is always less than the first Dirichlet eigenvalue, the situation is reversed for geodesic balls which are larger than a hemisphere (equality obtains at the hemisphere). For more discussion along these lines, the reader might consult [1], 8], [9, 14, and references therein.

As for extensions to bounded domains contained in $\mathbb{H}^{n}$, the situation is still unsettled, but we make the following comments and observations. The analog of Theorem 1.4 (the "naive" PPW conjecture for $\mathbb{H}^{n}$ ) cannot possibly hold, since one can imagine having a small disk (ball) with very narrow tentacles extending from it in such a way that the first two Dirichlet eigenvalues of this domain are very nearly those of the disk (ball), while the volume of the domain is as large as one wants. This means that $\Omega^{\star}$ can be an arbitrarily large ball, and the rub now comes from the fact that both eigenvalues of the large ball can be made arbitrarily close to the bottom of the spectrum of the Laplacian on all of $\mathbb{H}^{n}$, which is a positive value (specifically $\left.(n-1)^{2} / 4\right)$, by making the ball sufficiently large. This implies that $\lambda_{2} / \lambda_{1}\left(\Omega^{\star}\right)$ can be made as close as we want to 1 , while $\lambda_{2} / \lambda_{1}(\Omega)$ will stay close to its value for a small disk (ball). Since this latter value is nearly the Euclidean value (any value larger than 1 will do as well), we are faced with a contradiction. In fact, it is likely that $\lambda_{2} / \lambda_{1}$ for a geodesic ball in $\mathbb{H}^{n}$ is a decreasing function of the radius, i.e., that the counterpart to Theorem 1.3 goes the other way (this certainly appears to be the case for $\mathbb{H}^{2}$, based on numerical studies we have done). This, of course, would be an interesting fact to prove in its own right (and perhaps especially for its geometric implications), even if it doesn't lead into a proof of the "PPW conjecture for $\mathbb{H}^{n}$ ". It would also be, in a certain sense, the "natural" result, since $\lambda_{2} / \lambda_{1}$ is increasing for geodesic balls contained in a hemisphere of $\mathbb{S}^{n}$ (and quite possibly for all geodesic balls in $\mathbb{S}^{n}$; we note in this connection that $\lambda_{2} / \lambda_{1}$ goes to infinity as the full sphere is approached since in that limit $\lambda_{1} \rightarrow 0^{+}$while $\lambda_{2} \rightarrow n^{+}$), while it is constant for all balls in $\mathbb{R}^{n}$. As for an $\mathbb{H}^{n}$-analog of Theorem 1.1 (the "sophisticated" PPW conjecture for $\mathbb{H}^{n}$ ), this may well be true, but as yet it is not proved. One might also speculate that for bounded domains in $\mathbb{H}^{n} \lambda_{2} / \lambda_{1}$ is always less than the Euclidean bound (i.e., the value of $\lambda_{2} / \lambda_{1}$ for a Euclidean ball). This conjecture is certainly supported by the behavior of $\lambda_{2} / \lambda_{1}$ for geodesic balls in $\mathbb{H}^{n}$, and in general by the fact that "large" domains can be expected to have $\lambda_{2} / \lambda_{1}$ near 1. It is also supported by a result of Harrell and Michel [33, which gives a finite upper bound to $\lambda_{2} / \lambda_{1}$ for bounded domains contained in $\mathbb{H}^{2}$. While their bound is almost certainly not optimal, it is at least in the right ballpark: it is 17 , while the Euclidean value in two dimensions is approximately 2.5387 .

\section{ThE "CENTER OF MASS" RESUlT FOR DOMAINS IN SPHERES}

In this section we present a general Center of Mass Theorem which follows from general topological arguments. This Center of Mass Theorem guarantees the 
orthogonality of certain functions which is needed in later sections. In a previous paper [9] we gave such a theorem for domains contained in a hemisphere of $\mathbb{S}^{n}$. The proof of that theorem was fairly involved since we had to identify a hemisphere of $\mathbb{S}^{n}$ with the ball $B^{n}$ and use the Brouwer fixed point theorem. We also required a limiting argument since there were certain problems which arose for domains extending to the equator that we could not handle directly. These arguments would suffice to yield a version of Theorem 2.1 below for domains contained in a hemisphere (see Theorem 2.1 of [9] and Remark 4 following it to see how to make this extension). Such a result would be enough to allow us to prove only slightly weaker versions of the theorems found in the remainder of this paper. We have chosen to present the more general result here since its proof is both simpler and more natural than our former argument. In the new proof attention is confined to mappings from $\mathbb{S}^{n}$ to $\mathbb{S}^{n}$ and to certain natural geometrical conditions. A modest knowledge of degree theory is needed to conclude the proof.

Theorem 2.1 (Center of Mass Theorem). Let $\Omega$ be a domain in $\mathbb{S}^{n}$ and let $\tilde{G}$ be a continuous function on $[0, \pi]$ which is positive on $(0, \pi)$ and symmetric about $\pi / 2$. Also, let $d \mu$ be any positive measure on $\Omega$. Then there is a choice of Cartesian coordinates $x_{1}^{\prime}, x_{2}^{\prime}, \ldots, x_{n+1}^{\prime}$ for $\mathbb{R}^{n+1}$ with the origin at the center of $\mathbb{S}^{n}$ such that

$$
\int_{\Omega} x_{i}^{\prime} \tilde{G}(\theta) d \mu=0 \quad \text { for } i=1,2, \ldots, n,
$$

where $\theta$ represents the angle from the positive $x_{n+1}^{\prime}$-axis.

Remarks. In our later applications $d \mu$ will be $u_{1}^{2} d \sigma$ where $u_{1}$ is the first eigenfunction of $-\Delta$ on $\Omega$ with Dirichlet boundary conditions imposed on $\partial \Omega$ and $d \sigma$ is the standard volume element for $\mathbb{S}^{n}$. Similarly, in our applications the function $\tilde{G}$ will be related to the function $g(\theta)$ defined in equation (4.3) below (extended appropriately to $[0, \pi])$ by $\tilde{G}(\theta)=g(\theta) / \sin \theta$. Note that Theorem $[2.1$ makes no statement about the integral in (2.1) for the case $i=n+1$.

Proof. We begin by considering the vector function $\vec{v}: \mathbb{S}^{n} \rightarrow \mathbb{R}^{n+1}$ defined for $\vec{y} \in \mathbb{S}^{n}$ by

$$
\vec{v}(\vec{y})=\int_{\Omega} \vec{x} \tilde{G}(\theta) d \mu
$$

where $\theta$ represents the angle between $\vec{y}$ and the integration variable $\vec{x} \in \Omega$ and $\vec{x}=\left(x_{1}, x_{2}, \ldots, x_{n+1}\right)$ where $x_{1}, x_{2}, \ldots, x_{n+1}$ represent some (initial) set of Cartesian coordinates. The vector $\vec{v}(\vec{y})$ simply gives the center of mass in $\mathbb{R}^{n+1}$ of the hypersurface distribution on $\Omega \subset \mathbb{S}^{n}$ with mass density given by $\tilde{G}(\theta) d \mu$. Note that its dependence on the point $\vec{y}$ is entirely through the function $\tilde{G}(\theta)$; indeed, if not for this function we would have only a single center of mass vector $\vec{v}$.

First we argue that what we should look for are points $\vec{y}_{0} \in \mathbb{S}^{n}$ such that

$$
\vec{v}\left(\vec{y}_{0}\right)=\alpha \vec{y}_{0},
$$

i.e., such that $\vec{y}_{0}$ and $\vec{v}\left(\vec{y}_{0}\right)$ are linearly dependent. To see that finding such a point will suffice to prove our theorem, suppose $\vec{y}_{0}$ is a point where (2.3) holds and let $R$ be an $(n+1)$ by $(n+1)$ rotation matrix with $\vec{y}_{0}$ as its last row. Defining new 
Cartesian coordinates $x_{1}^{\prime}, x_{2}^{\prime}, \ldots, x_{n+1}^{\prime}$ via

$$
x_{i}^{\prime}=\sum_{j=1}^{n+1} R_{i j} x_{j}
$$

we have (with $\theta$ measured from $\vec{y}_{0}$ )

$$
\begin{aligned}
\int_{\Omega} x_{i}^{\prime} \tilde{G}(\theta) d \mu & =\sum_{j=1}^{n+1} R_{i j} \int_{\Omega} x_{j} \tilde{G}(\theta) d \mu=\left[R \vec{v}\left(\vec{y}_{0}\right)\right]_{i}=\left[\alpha R \vec{y}_{0}\right]_{i} \\
& =\left[\alpha \hat{e}_{n+1}\right]_{i}=0 \quad \text { for } i=1, \ldots, n,
\end{aligned}
$$

since $R$ is an orthogonal matrix and hence its first $n$ rows are orthogonal to its last row. Thus, the conclusion to the theorem will follow from finding a solution to (2.3) and from here on we concentrate on finding such a solution.

Now if $\vec{v}(\vec{y})$ ever vanished for some $\vec{y} \in \mathbb{S}^{n}$, the conclusion (2.1) would follow immediately with no need to rotate coordinates. So we may as well assume that $\vec{v}$ never vanishes on $\mathbb{S}^{n}$. Under this assumption we can pass to consideration of the mapping $\vec{w}: \mathbb{S}^{n} \rightarrow \mathbb{S}^{n}$ defined by

$$
\vec{w}(\vec{y})=\frac{\vec{v}(\vec{y})}{|\vec{v}(\vec{y})|} .
$$

The dependence condition (2.3) then reduces to

$$
\vec{w}\left(\vec{y}_{0}\right)= \pm \vec{y}_{0}
$$

so that we are seeking a fixed point or an "anti-fixed point" of $\vec{w}$. Under the assumptions of the theorem it suffices to seek only fixed points, i.e., solutions to

$$
\vec{w}\left(\vec{y}_{0}\right)=\vec{y}_{0} .
$$

This follows from the symmetry of $\tilde{G}(\theta)$ about $\theta=\pi / 2$ which implies that $\vec{v}(-\vec{y})=$ $\vec{v}(\vec{y})$ for all $\vec{y} \in \mathbb{S}^{n}$ and thus that $\vec{w}(-\vec{y})=\vec{w}(\vec{y})$ for all $\vec{y} \in \mathbb{S}^{n}$. Hence if $\vec{y}_{0}$ is a solution to (2.7), then either $\vec{y}_{0}$ or $-\vec{y}_{0}$ must be a solution to (2.8) and we can therefore concentrate solely on finding solutions to 2.8). (Geometrically, too, it is more natural to view a point $\vec{y}_{0}$ satisfying (2.8) as a center of mass of $\Omega$ than the point $-\vec{y}_{0}$ satisfying $\vec{w}\left(-\vec{y}_{0}\right)=\vec{y}_{0}$, even though there is no difference between the two as far as fulfilling the conditions of the theorem goes.)

Finally, we are at a point where we can use degree theory to conclude that $\vec{w}$ must have a fixed point, i.e., a solution $\vec{y}_{0}$ to (2.8). The definition of $\vec{v}$ shows that it is continuous and our assumption that $\vec{v}$ does not vanish on $\mathbb{S}^{n}$ guarantees the continuity of $\vec{w}$ as defined by (2.6). Therefore, by standard theory (see, for example, 2], p. 195; 29], Chapter 16, Section 1; 35], p. 263; [40, p. 116; [47, Chapter 16; [53], p. 807) $\vec{w}$ has a degree as a map from $\mathbb{S}^{n}$ to $\mathbb{S}^{n}$. The fact that $\vec{w}(-\vec{y})=\vec{w}(\vec{y})$ for all $\vec{y} \in \mathbb{S}^{n}$ implies that $\operatorname{deg}(\vec{w})$ is even (simply observe that for an image point $\vec{z} \in \mathbb{S}^{n}$ the points in the preimage come in pairs $\pm \vec{y}$ ). Now if $\vec{w}$ has no fixed points, i.e., if $\vec{w}(\vec{y}) \neq \vec{y}$ for all $\vec{y} \in \mathbb{S}^{n}$, then $\vec{w}$ would be homotopic to the antipodal map $\vec{A}$ defined by $\vec{A}(\vec{y})=-\vec{y}$ via the homotopy

$$
h(t, \vec{y})=\frac{-t \vec{y}+(1-t) \vec{w}(\vec{y})}{|-t \vec{y}+(1-t) \vec{w}(\vec{y})|} \quad \text { for } 0 \leq t \leq 1 .
$$


But the degree of a mapping is a homotopy invariant and $\operatorname{deg}(\vec{A})= \pm 1 \neq \operatorname{deg}(\vec{w})$, a contradiction. Hence $\vec{w}$ must have some fixed point $\vec{y}_{0} \in \mathbb{S}^{n}$ and the proof is complete.

Remarks. (1) If $\Omega$ is contained in a hemisphere of $\mathbb{S}^{n}$, then another way to complete the proof of the theorem is to set things up initially in a Cartesian frame such that $\Omega$ lies in the northern hemisphere of $\mathbb{S}^{n}$ and observe that for any $\vec{y} \in \mathbb{S}^{n}$,

$$
v_{n+1}(\vec{y})=\int_{\Omega} x_{n+1} \tilde{G}(\theta) d \mu>0 .
$$

Hence $\vec{v}$ never vanishes and we may regard $\vec{w}=\vec{v} /|\vec{v}|$ as a mapping from the closed northern hemisphere into itself. Since this space is homeomorphic to the ball $B^{n}$, we can apply the Brouwer fixed point theorem to conclude that $\vec{w}$ has a fixed point $\vec{y}_{0}$ in the northern hemisphere (see, for example, [39., Sections 8-10; [47], p. 406; [48], pp. 151, 194). This proof is similar to, but simpler than, the proof of the restricted version of Theorem 2.1 that we gave in 9.

(2) The alternative proof just given does not use the symmetry of $\tilde{G}(\theta)$ about $\theta=\pi / 2$ stated in the theorem. Only positivity of $\tilde{G}(\theta)$ on $(0, \pi)$ is used. There are certainly situations, in particular, for domains which are in some sense larger than a hemisphere, where one might not want to require that $\tilde{G}$ be symmetric about $\pi / 2$. In such situations one possible route to a center of mass result is to show that the mapping $\vec{w}$ misses at least one point of $\mathbb{S}^{n}$, following the spirit of the alternative proof given above. Brouwer's fixed point theorem can then be applied (to $\mathbb{S}^{n}$ less a sufficiently small neighborhood of a point that $\vec{w}$ misses) to yield a fixed point. Or in the language of degree theory, the case where a map misses a point is the simplest case of a map which has degree 0 . This can be seen directly or by observing that such a map $\vec{w}$ is homotopic to a constant map, i.e., contractible to a point, or inessential (see 35], p. 154; 39], p. 357; or [48, p. 23) via the homotopy

$$
g(t, \vec{y})=\frac{-t \vec{z}_{0}+(1-t) \vec{w}(\vec{y})}{\left|-t \vec{z}_{0}+(1-t) \vec{w}(\vec{y})\right|} \quad \text { for } 0 \leq t \leq 1
$$

if $\vec{z}_{0}$ is a point that $\vec{w}$ misses.

(3) If $\Omega$ has a center of symmetry and $d \mu$ is either $u_{1}^{2} d \sigma$ (as occurs in Dirichlet problems for $-\Delta$ on $\Omega$ ) or $d \sigma$ (as occurs in Neumann problems for $-\Delta$ on $\Omega$ ), then this point will certainly serve as a center of mass in the sense of Theorem 2.1 Here $d \sigma$ represents the standard volume element for $\mathbb{S}^{n}$ and $u_{1}$ represents the first Dirichlet eigenfunction of $-\Delta$ on $\Omega$. One has only to note that $u_{1}$, being unique up to a constant factor and of one sign, must share the symmetries of $\Omega$. Also, if $d \mu=d \sigma$ and $\Omega$ is an arbitrary domain such that its complement has a center of mass in the sense of Theorem 2.1] (as could be concluded, for example, via any of the conditions discussed so far, or by other means), then $\Omega$ shares this center of mass since it is clear that

$$
\int_{\mathbb{S}^{n}} x_{i}^{\prime} \tilde{G}(\theta) d \sigma=0 \quad \text { for } i=1,2, \ldots, n
$$

if $x_{1}^{\prime}, \ldots, x_{n+1}^{\prime}$ represent Cartesian coordinates and $\theta$ is measured from the positive $x_{n+1}^{\prime}$-axis. In particular, $\Omega$ certainly has a center of mass in this sense if the complement of $\Omega$ is contained in a hemisphere. All the observations made in this remark apply whether or not $\tilde{G}(\theta)$ is even with respect to $\theta=\pi / 2$. For another result that holds in the absence of symmetry of $\tilde{G}$ about $\pi / 2$ see Theorem 2.2 below. 
(4) With Theorem 2.1 in hand we can obtain a modest improvement of our main theorem in [9] (see Theorem 5.1). In particular, in Remark 2 following the proof of Theorem 5.1 in $[9$ we now have no need to invoke condition (2.4). We thus obtain the result $\mu_{1}(\Omega) \leq \mu_{1}\left(\Omega^{\star}\right)$ comparing the first nonzero Neumann eigenvalue of the domain $\Omega$ with that of the spherical cap $\Omega^{\star}$ having the same volume for any domain $\Omega$ such that $\Omega \cap(-\Omega)=\emptyset$ (equivalently, $-\Omega \subset \mathbb{S}^{n} \backslash \Omega$ ). This inequality is an equality if and only if $\Omega$ is itself a geodesic ball. More generally, the same result holds if, when the north pole is a center of mass in the sense of Theorem 2.1, $\Omega$ has the property that for each $k \in[0,1]$

$$
\left|\left\{\vec{y} \in \Omega \mid y_{n+1} \leq-k\right\}\right| \leq\left|\left\{\vec{y} \in \mathbb{S}^{n} \backslash \Omega \mid y_{n+1} \geq k\right\}\right|
$$

(cf. Remark 2 following the proof of Theorem 5.1 in [9]). Here $|X|$ denotes the measure of $X$ with respect to the canonical measure (standard volume element) on $\mathbb{S}^{n}$ where $X$ is any measurable subset of $\mathbb{S}^{n}$. We refer to condition (2.13) as the "excess less than or equal to deficit property". For some further comments relating to this property, see our remarks at the end of Section 6 . These give the most general conditions known at this time.

Finally, for possible future use (see also Remarks 2 and 3 above) we state the following variant of our Center of Mass Theorem which holds in even dimension (i.e., for $\Omega \subset \mathbb{S}^{n}$ with $n$ even) in the absence of symmetry of $\tilde{G}$ about $\pi / 2$ :

Theorem 2.2. Let $\Omega$ be a domain in $\mathbb{S}^{n}$ for $n$ even and let $\tilde{G}$ be continuous on $[0, \pi]$ and positive on $(0, \pi)$. Then $\Omega$ has a center of mass in the sense of Theorem 2.1. That is, there exists a choice of Cartesian coordinates such that (2.1) holds for $i=1,2, \ldots, n$.

Proof. Defining $\vec{v}$ as above (equation (2.2)) either $\vec{v}$ vanishes somewhere and we are done or we can pass to $\vec{w}=\vec{v} /|\vec{v}|$. Continuing with the latter case, if $\vec{w}$ has neither a fixed point nor an anti-fixed point (i.e., there are no solutions $\vec{y} \in \mathbb{S}^{n}$ to $\vec{w}(\vec{y})= \pm \vec{y}$ ), then as above we can show that $\vec{w}$ is homotopic to the antipodal map and also to the identity map. But the antipodal map has degree $(-1)^{n+1}$ (see, for example, 2], p. 197, Theorem 9.2; 29], p. 339, Exercise 4; [40], p. 118, Theorem 21.3 ; [4], p. 403, Theorem 4.3; 53], p. 809) and for $n$ even $(-1)^{n+1}=-1 \neq 1=$ degree of the identity map. This is a contradiction since degree is a homotopy invariant (see [2], p. 195; 29], p. 339; 35], p. 266; 40], p. 117; [47, p. 401; or [53], p. 809), hence $\vec{w}$ must have either a fixed point or an anti-fixed point, and the conclusion of the theorem follows.

\section{Properties of the first two Dirichlet eigenvalues OF GEODESIC BALLS IN $\mathbb{S}^{n}$}

We consider the Dirichlet problem on a geodesic ball of radius $\theta_{1}$ in $\mathbb{S}^{n}$ (where $\left.\theta_{1} \in(0, \pi)\right)$ which we view as a polar cap centered at the north pole (i.e., the point $\hat{e}_{n+1} \in \mathbb{S}^{n}$ is taken as the center of our geodesic ball). By using the $O(n)$ symmetry of the polar cap (this $O(n)$ is the subgroup of $O(n+1)$ which leaves the point $\hat{e}_{n+1}$ fixed), one can separate variables in the usual way obtaining the family of ordinary differential equations in the "radial" variable $\theta$

$$
-y^{\prime \prime}-(n-1) \cot \theta y^{\prime}+m(m+n-2) \csc ^{2} \theta y=\lambda y \quad \text { on }\left(0, \theta_{1}\right)
$$

for $m=0,1,2, \ldots$ The boundary conditions to be applied for (3.1) are $y(0)$ finite and $y\left(\theta_{1}\right)=0$. In particular, we shall be concerned with the lowest eigenvalues of 
the $m=0$ and $m=1$ cases of this equation, but first we develop some general properties of the solutions to these equations and some of their interrelationships.

We begin by considering $\lambda$ as a positive parameter (all the eigenvalues that we consider here are easily seen to be positive by consideration of the Rayleigh quotients that characterize them) and defining $u_{m}(\theta ; \lambda)$ for $m=0,1,2, \ldots$ as that solution to (3.1) which has the behavior

$$
u_{m}(\theta ; \lambda)=c_{m} \theta^{m}+O\left(\theta^{m+2}\right)
$$

where the constants $c_{m}$ will be specified below. This behavior is consistent with equation (3.1) as can be seen from Frobenius theory $(\theta=0$ is a regular singular point of (3.1)). In particular, the eigenfunctions to (3.1) will all be found among the $u_{m}$ 's defined in (3.2) assuming $c_{m} \neq 0$ since finiteness at $\theta=0$ forces this behavior (up to multiplicative factors). Moreover, it is not difficult to verify that if $u_{m}$ solves (3.1), then

$$
u_{m}^{\prime}-m \cot \theta u_{m}
$$

satisfies (3.1) for $m$ replaced by $m+1$ and also

$$
u_{m}^{\prime}+(m+n-2) \cot \theta u_{m}
$$

satisfies (3.1) for $m$ replaced by $m-1$. From these facts and Frobenius theory again it follows that

$$
u_{m+1}=-u_{m}^{\prime}+m \cot \theta u_{m}
$$

and

$$
[\lambda-(m-1)(m+n-2)] u_{m-1}=u_{m}^{\prime}+(m+n-2) \cot \theta u_{m}
$$

if we agree to set $c_{0}=1$ and define successive $c_{m}$ 's via

$$
c_{m+1}=\frac{\lambda-m(m+n-1)}{2 m+n} c_{m} \quad \text { for } m=0,1,2, \ldots
$$

These constitute the raising and lowering relations for the functions $u_{m}$. Also, elimination of $u_{m}^{\prime}$ between (3.3) and (3.4) yields the pure recursion relation in $m$

$$
u_{m+1}-(2 m+n-2) \cot \theta u_{m}+[\lambda-(m-1)(m+n-2)] u_{m-1}=0 .
$$

Since $(\sin \theta)^{m+n-2} u_{m}(\theta)$ is 0 at $\theta=0$ one can integrate (3.4) and replace $m$ by $m+1$ to obtain

$$
(\sin \theta)^{m+n-1} u_{m+1}(\theta)=[\lambda-m(m+n-1)] \int_{0}^{\theta}(\sin t)^{m+n-1} u_{m}(t) d t .
$$

What we have developed so far could be considered the algebraic properties of the functions $u_{m}$. One should think of the $u_{m}$ 's as the analogs of associated Legendre functions in $n$ dimensions. In particular, when $n=2$ one finds that

$$
u_{m}(\theta ; \nu(\nu+1))=(-1)^{m} P_{\nu}^{m}(\cos \theta)
$$

following [1] (in general this should be correct up to a constant factor, typically a factor of $(-1)^{m}$, depending on the precise convention adopted; cf. [46, for example, where the convention differs by $\left.(-1)^{m}\right)$; here we have replaced the eigenvalue parameter $\lambda$ by $\nu(\nu+1)$, as is traditional in dealing with Legendre functions. In this case (3.1) reduces to the associated Legendre equation and (3.3), (3.4), and (3.6) all reduce to standard relations between the associated Legendre functions. Almost certainly this generalization to $n$ dimensions of associated Legendre functions and their basic relations has been developed before, though we do not know 
of a reference where the details needed here are developed explicitly. (Cf. also 31] which deals with the $m=0$ case of (3.1) in $n$ dimensions but in Schrödinger normal form.) In any event, it is a relatively simple matter to pass to the $n$-dimensional case once the situation in two dimensions is known. In fact, our functions $u_{m}$ can be expressed in terms of associated Legendre functions no matter what the dimension but this connection is not particularly useful in this context so we do not elaborate upon it here (but see the equations in Section 6 following equation (6.4), or Remark 1 below). We note that our convention on the constants $c_{m}$ allows them to vanish from a certain value of $m$ on for specific values of the parameter $\lambda$ (cf. (3.5). (Recall, for example, that when dealing with the full sphere $\mathbb{S}^{2}$ one needs $P_{\ell}^{m}$ only for $\ell=0,1, \ldots$ and $m=0,1, \ldots, \ell$.) This is not a problem for our purposes here since we will be most interested in passing from $u_{m}$ to $u_{m+1}$ via either (3.3) or (3.7).

We come back now to the eigenvalue problem for (3.1). If one defines the lefthand side of equation (3.1) as the operator $h_{m}$ applied to $y$ (with boundary conditions incorporated in the definition of $h_{m}$ ), then it is easily seen that $h_{m^{\prime}}>h_{m}$ in the sense of quadratic forms if $m^{\prime}>m$. Thus $\lambda_{1}$ of $-\Delta$ for the polar cap is $\lambda_{1}\left(h_{0}\right)$ while $\lambda_{2}$ of $-\Delta$ for the polar cap must be either $\lambda_{1}\left(h_{1}\right)$ or $\lambda_{2}\left(h_{0}\right)$. We now show that the former is the case.

Lemma 3.1. The first eigenvalue of the Dirichlet Laplacian on a polar cap is the first eigenvalue of (3.1) with $m=0$ while the second eigenvalue of the Dirichlet Laplacian is the first eigenvalue of (3.1) with $m=1$. The second eigenvalue of the cap occurs with multiplicity $n$. These results hold for all polar caps, i.e., for all $\theta_{1} \in(0, \pi)$.

Proof. In the notation of the preceding paragraph we must show that $\lambda_{2}\left(h_{0}\right)>$ $\lambda_{1}\left(h_{1}\right)$. The argument proceeds via a simple use of Rolle's theorem as applied to (3.3) and (3.4) rewritten in the forms

$$
\left[(\sin \theta)^{-m} u_{m}\right]^{\prime}=-(\sin \theta)^{-m} u_{m+1}
$$

and

$$
\left[(\sin \theta)^{m+n-2} u_{m}\right]^{\prime}=[\lambda-(m-1)(m+n-2)](\sin \theta)^{m+n-2} u_{m-1} .
$$

In particular, with $m=0$ in the first of these we have

$$
u_{0}^{\prime}=-u_{1}
$$

and with $m=1$ in the second we have

$$
\left[(\sin \theta)^{n-1} u_{1}\right]^{\prime}=\lambda(\sin \theta)^{n-1} u_{0} .
$$

By Rolle's theorem, between any two zeros of $u_{0}$ there is a zero of $u_{0}^{\prime}$ and hence of $u_{1}$, since (3.10) holds. Similarly, between two zeros of $(\sin \theta)^{n-1} u_{1}$ there is a zero of its derivative and hence, by (3.11), of $u_{0}$. Thus for fixed $\lambda>0$ the zeros of $u_{0}$ and $(\sin \theta)^{n-1} u_{1}$ on $[0, \pi)$ interlace.

Now consider $u_{0}$ and $u_{1}$ for $\lambda=\lambda_{1}\left(h_{1}\right)$. Since this makes $\theta_{1}$ the first positive zero of $u_{1}$ it is clear by what we have just proved that $u_{0}$ has exactly one zero in $\left(0, \theta_{1}\right)$ and that $\theta_{1}$ is not a zero of $u_{0}$. This then implies, by the fact that the positive zeros of any $u_{m}$ are decreasing functions of the parameter $\lambda$ (see, for example, [20], p. 315 , or [28], p. 454), that $\lambda_{2}\left(h_{0}\right)>\lambda_{1}\left(h_{1}\right)$.

That the multiplicity of $\lambda_{1}\left(h_{1}\right)$ as an eigenvalue of $-\Delta$ on the polar cap (= geodesic ball) with Dirichlet boundary conditions is $n$ follows from the details of separation of variables. It can be shown that the corresponding eigenfunctions can 
be taken as $\left(x_{i} / \sin \theta\right) y(\theta)$ (restricted to $\left.\mathbb{S}^{n}\right)$ where $i=1,2, \ldots, n, x_{n+1}=\cos \theta$, and $y(\theta)$ is the eigenfunction of (3.1) for the eigenvalue $\lambda_{1}\left(h_{1}\right)$. These functions form an orthogonal basis for the eigenspace of $-\Delta$ corresponding to the eigenvalue $\lambda_{1}\left(h_{1}\right)$, showing that its multiplicity is $n$. This completes our proof.

Remarks. (1) The argument used in our proof above can be viewed as a way of translating order properties of the zeros of the $u_{m}$ 's into order properties of the corresponding eigenvalues $\lambda_{i}\left(h_{m}\right)$. In fact, our approach extends easily to an interlacing result for the zeros (and hence for the associated eigenvalues) of $u_{m}$ and $u_{m+1}$ for arbitrary $m$. Further ordering properties of the Dirichlet eigenvalues of spherical caps in $\mathbb{S}^{n}$, at least for even $n$ (and surely the case of odd $n$ could be handled similarly), may be inferred from the papers of Baginski [12], [13]. We note, though, that all of Baginski's results are presented in terms of the zeros of the associated Legendre functions $P_{\nu}^{m}$ in the variable $\nu$ (for $m$ an integer). To make the connection to the present setting, one makes use of the formulas in Section 6 following equation (6.4) (or equations (3.17) and (3.18) in 8], with the correction that the upper index in both associated Legendre functions should be negated), which relate our functions $u_{m}$ as defined above to associated Legendre functions (up to constant factors). Note, in this connection, that $y_{1}(\theta)=u_{0}\left(\theta ; \lambda_{1}\right)$ and $y_{2}(\theta)=u_{1}\left(\theta ; \lambda_{2}\right)$ up to constant factors (as proved in Lemma 3.1, throughout this section we take these factors to be 1). In general, one has $u_{m}(\theta ; \lambda)$ proportional to $(\sin \theta)^{1-n / 2} P_{\nu}^{-(n / 2-1+m)}(\cos \theta)$, where $\lambda$ and $\nu$ are related by $\lambda=(\nu-n / 2+1)(\nu+n / 2)$. We thank the referee for calling Baginski's papers to our attention.

(2) Another proof of Lemma 3.1 follows by mimicking our proof of Lemma 3.1 of [9. With $\tau=\lambda_{2}\left(h_{0}\right)$ and $v$ as the associated eigenfunction we can assume that for some $a \in\left(0, \theta_{1}\right) v>0$ on $(0, a)$ and $v<0$ on $\left(a, \theta_{1}\right)$, and hence that $v(a)=0$, $v^{\prime}(a)<0$. Also we take $\lambda=\lambda_{1}\left(h_{1}\right)$ and set $g=u_{1}(\theta ; \lambda)$ and $h=u_{0}(\theta ; \lambda)$. Since $c_{1}=\lambda / n>0$ it is clear that $g>0$ on $\left(0, \theta_{1}\right)$ and that $g(0)=0=g\left(\theta_{1}\right)$. It also follows that $g=-h^{\prime}$ and $h$ satisfies

$$
-h^{\prime \prime}-(n-1) \cot \theta h^{\prime}=\lambda h
$$

while $v$ satisfies

$$
-v^{\prime \prime}-(n-1) \cot \theta v^{\prime}=\tau v .
$$

From the last two equations we obtain

$$
\left[(\sin \theta)^{n-1}\left(v h^{\prime}-v^{\prime} h\right)\right]^{\prime}=-(\lambda-\tau)(\sin \theta)^{n-1} v h
$$

and integration from $a$ to $\theta_{1}$ produces

$$
\left(\sin \theta_{1}\right)^{n-1} v^{\prime}\left(\theta_{1}\right) h\left(\theta_{1}\right)-(\sin a)^{n-1} v^{\prime}(a) h(a)=(\lambda-\tau) \int_{a}^{\theta_{1}} v h \sin ^{n-1} \theta d \theta
$$

We now argue by contradiction, so assume $\tau \leq \lambda$. Since $v(a)=0, v=u_{0}(\theta ; \tau)$, $h=u_{0}(\theta ; \lambda)$ and $\tau \leq \lambda$, by the fact that the positive zeros of $u_{0}(\theta ; \lambda)$ are decreasing with increasing $\lambda$ it follows that the first positive zero of $h$ is less than or equal to $a$ and, since $h^{\prime}=-g<0$ on $\left(0, \theta_{1}\right)$, it therefore follows that $h<0$ on $\left(a, \theta_{1}\right]$. But now (3.12) gives a contradiction, since its right-hand side is greater than or equal to 0 while its left-hand side is negative (note that $v^{\prime}(a)<0$ and $v^{\prime}\left(\theta_{1}\right)>0$ since $a$ and $\theta_{1}$ must be successive zeros of $v$ ).

(3) Yet another proof of Lemma 3.1 would be via the level-ordering results of Baumgartner, Grosse, and Martin [18], [19]. Specifically, see our papers [3], [4] 
where proofs for a ball in $\mathbb{R}^{n}$ occur and also the papers [16, [17] of Baumgartner, which give extensions to cases arising from separation of variables in spherical coordinates in spaces of constant curvature.

For future reference we note the following lemma.

Lemma 3.2. If $0<\theta_{1}<\pi$, the first eigenfunction of (3.1) with $m=0$, i.e., $y_{1}(\theta) \equiv u_{0}\left(\theta ; \lambda_{1}\right)$, is strictly decreasing on $\left[0, \theta_{1}\right]\left(y_{1}>0\right.$ on $\left[0, \theta_{1}\right)$ is our convention for $y_{1}$ here and throughout this paper; this follows from our choice $c_{0}=1$ ).

Proof. $u_{0}\left(\theta ; \lambda_{1}\right)$ satisfies

$$
-\left(\sin ^{n-1} \theta u_{0}^{\prime}\right)^{\prime}=\lambda_{1}\left(\theta_{1}\right) \sin ^{n-1} \theta u_{0}>0
$$

in $\left[0, \theta_{1}\right)$ (since $\lambda_{1}(\Omega)>0$ follows from the variational characterization of the eigenvalues of $-\Delta$ via the Rayleigh quotient $\left.\int_{\Omega}|\nabla \varphi|^{2} / \int_{\Omega} \varphi^{2}\right)$, which implies that $\sin ^{n-1} \theta u_{0}^{\prime}$ is decreasing in $\left[0, \theta_{1}\right)$. Hence $\sin ^{n-1} \theta u_{0}^{\prime}<\left.\left(\sin ^{n-1} \theta u_{0}^{\prime}\right)\right|_{\theta=0}=0$, which proves the lemma.

Having identified $\lambda_{1}$ and $\lambda_{2}$ for $-\Delta$ on a spherical cap with Dirichlet boundary conditions we are now in a position to investigate their behaviors and, in particular, that of $\lambda_{2} / \lambda_{1}$. Since our concern will be with how these functions vary with $\theta_{1}$, the geodesic radius of the spherical cap, we shall denote $\lambda_{1}$ and $\lambda_{2}$ by $\lambda_{1}\left(\theta_{1}\right)$ and $\lambda_{2}\left(\theta_{1}\right)$ throughout the remainder of this section. Associated with equation (3.1) is the one-dimensional Schrödinger operator

$$
H_{m}\left(\theta_{1}\right)=-\frac{d^{2}}{d \theta^{2}}+\frac{(2 m+n-1)(2 m+n-3)}{4 \sin ^{2} \theta}-\frac{(n-1)^{2}}{4}
$$

acting on $L^{2}\left(\left(0, \theta_{1}\right), d \theta\right)$ with Dirichlet boundary conditions imposed at 0 and $\theta_{1}$. The operators $H_{m}\left(\theta_{1}\right)$ form a family of self-adjoint operators. It is clear by Lemma 3.1 that $\lambda_{1}\left(\theta_{1}\right)=\lambda_{1}\left(H_{0}\left(\theta_{1}\right)\right)$ and $\lambda_{2}\left(\theta_{1}\right)=\lambda_{1}\left(H_{1}\left(\theta_{1}\right)\right)$.

We now analyze how $\lambda_{1}$ and $\lambda_{2}$ vary with $\theta_{1}$ by using perturbation theory [34, [36], [45]. To be successful at this we need to work on a fixed interval $\left(0, \theta_{1}\right)$ and we do this by observing that the eigenvalue problem $H_{m}\left(c \theta_{1}\right) v=\lambda v$ on $\left(0, c \theta_{1}\right)$ can be rescaled to

$$
\left[-\frac{1}{c^{2}} \frac{d^{2}}{d t^{2}}+\frac{(2 m+n-1)(2 m+n-3)}{4 \sin ^{2} c t}-\frac{(n-1)^{2}}{4}\right] v=\lambda v \quad \text { for } t \in\left(0, \theta_{1}\right)
$$

which is equivalent to

$$
\left[-\frac{d^{2}}{d \theta^{2}}+\frac{(2 m+n-1)(2 m+n-3) c^{2}}{4 \sin ^{2} c \theta}-\frac{(n-1)^{2} c^{2}}{4}\right] v=c^{2} \lambda v \quad \text { for } \theta \in\left(0, \theta_{1}\right) \text {. }
$$

As was done above for $H_{m}\left(\theta_{1}\right)$, we define an operator $\tilde{H}_{m}(c)$ on $L^{2}\left(0, \theta_{1}\right)$ via the differential expression appearing on the left-hand side of (3.15). It is clear from (3.15) $)$ that $\lambda_{k}\left(\tilde{H}_{m}(c)\right)=c^{2} \lambda_{k}\left(H_{m}\left(c \theta_{1}\right)\right)$ and thus, in particular, that

$$
\lambda_{1}\left(c \theta_{1}\right)=c^{-2} \lambda_{1}\left(\tilde{H}_{0}(c)\right)
$$

and

$$
\lambda_{2}\left(c \theta_{1}\right)=c^{-2} \lambda_{1}\left(\tilde{H}_{1}(c)\right)
$$


What we intend to do now is to determine the derivatives $\lambda_{1}^{\prime}\left(\theta_{1}\right)$ and $\lambda_{2}^{\prime}\left(\theta_{1}\right)$ using perturbation theory and the fact that

$$
\lambda_{j}^{\prime}\left(\theta_{1}\right)=\left.\frac{1}{\theta_{1}} \frac{d \lambda_{j}\left(c \theta_{1}\right)}{d c}\right|_{c=1} .
$$

Since $\tilde{H}_{m}(c)$ is an analytic family in $c$ for $c$ near 1 we can apply regular RayleighSchrödinger perturbation theory [34, [36], 45]. In fact

$$
\begin{gathered}
\tilde{H}_{m}(c)=H_{m}\left(\theta_{1}\right)+\frac{(2 m+n-1)(2 m+n-3)}{4}\left(\frac{c^{2}}{\sin ^{2} c \theta}-\frac{1}{\sin ^{2} \theta}\right) \\
-\frac{(n-1)^{2}\left(c^{2}-1\right)}{4}=H_{m}\left(\theta_{1}\right)+V_{m}(\theta ; c)
\end{gathered}
$$

and since $V_{m}(\theta ; c)$ is analytic in $c$ for $c$ near 1 we are assured that the operators $\tilde{H}_{m}(c)$ form an analytic family of type (A) for $c$ near 1 (see [45], p. 16 for the definition of analytic family of type (A), or see [34, p. 154; also see Chapter 7 of 36 for the definitive account of analytic perturbation theory). This allows us to compute the derivatives of the eigenvalues of $\tilde{H}_{m}(c)$ using the first-order perturbation formula (cf. Kato [36], p. 391, eq. (3.18))

$$
\left.\frac{d \lambda_{1}\left(\tilde{H}_{m}(c)\right)}{d c}\right|_{c=1}=\frac{\int_{0}^{\theta_{1}}\left[\left.\frac{\partial V_{m}}{\partial c}(\theta ; c)\right|_{c=1}\right] v_{m}^{2} d \theta}{\int_{0}^{\theta_{1}} v_{m}^{2} d \theta}
$$

where the functions $v_{m}(\theta)$ denote first eigenfunctions of $H_{m}\left(\theta_{1}\right)=\tilde{H}_{m}(1)$.

By (3.18) we have

$$
\begin{aligned}
\frac{d}{d \theta_{1}} & {\left[\frac{\lambda_{2}\left(\theta_{1}\right)}{\lambda_{1}\left(\theta_{1}\right)}\right]=\left.\frac{1}{\theta_{1}}\left[\frac{d}{d c}\left(\frac{\lambda_{2}\left(c \theta_{1}\right)}{\lambda_{1}\left(c \theta_{1}\right)}\right)\right]\right|_{c=1} } \\
& =\frac{1}{\theta_{1}}\left[\lambda_{1}\left(\theta_{1}\right)\left(\left.\frac{d}{d c} \lambda_{2}\left(c \theta_{1}\right)\right|_{c=1}\right)-\lambda_{2}\left(\theta_{1}\right)\left(\left.\frac{d}{d c} \lambda_{1}\left(c \theta_{1}\right)\right|_{c=1}\right)\right] \frac{1}{\lambda_{1}\left(\theta_{1}\right)^{2}} .
\end{aligned}
$$

Thus, showing that $\lambda_{2}\left(\theta_{1}\right) / \lambda_{1}\left(\theta_{1}\right)$ increases with increasing $\theta_{1}$ comes down to showing that

$$
0<\frac{1}{\lambda_{2}\left(\theta_{1}\right)}\left(\left.\frac{d}{d c} \lambda_{2}\left(c \theta_{1}\right)\right|_{c=1}\right)-\frac{1}{\lambda_{1}\left(\theta_{1}\right)}\left(\left.\frac{d}{d c} \lambda_{1}\left(c \theta_{1}\right)\right|_{c=1}\right),
$$

which, by equations (3.16) and (3.17), reduces to showing

$$
0<\frac{1}{\lambda_{2}\left(\theta_{1}\right)}\left(\left.\frac{d}{d c} \lambda_{1}\left(\tilde{H}_{1}(c)\right)\right|_{c=1}\right)-\frac{1}{\lambda_{1}\left(\theta_{1}\right)}\left(\left.\frac{d}{d c} \lambda_{1}\left(\tilde{H}_{0}(c)\right)\right|_{c=1}\right) .
$$

From (3.19) we obtain

$$
\left.\frac{\partial V_{m}}{\partial c}(\theta ; c)\right|_{c=1}=\frac{1}{2}(2 m+n-1)(2 m+n-3) \csc ^{2} \theta(1-\theta \cot \theta)-\frac{1}{2}(n-1)^{2}
$$

so that, by (3.20),

$$
\left.\frac{d}{d c} \lambda_{1}\left(\tilde{H}_{1}(c)\right)\right|_{c=1}=\frac{1}{2}(n-1) \frac{\int_{0}^{\theta_{1}}\left[(n+1) \csc ^{2} \theta(1-\theta \cot \theta)-(n-1)\right] v_{1}^{2} d \theta}{\int_{0}^{\theta_{1}} v_{1}^{2} d \theta},
$$


and

$$
\left.\frac{d}{d c} \lambda_{1}\left(\tilde{H}_{0}(c)\right)\right|_{c=1}=\frac{1}{2}(n-1) \frac{\int_{0}^{\theta_{1}}\left[(n-3) \csc ^{2} \theta(1-\theta \cot \theta)-(n-1)\right] v_{0}^{2} d \theta}{\int_{0}^{\theta_{1}} v_{0}^{2} d \theta}
$$

The functions $v_{0}$ and $v_{1}$ are related to $u_{0}\left(\theta ; \lambda_{1}\right)$ and $u_{1}\left(\theta ; \lambda_{2}\right)$ by $v_{0}=u_{0} \sin ^{(n-1) / 2} \theta$ and $v_{1}=u_{1} \sin ^{(n-1) / 2} \theta$ respectively.

Introducing the functions $\ell(\theta)=\cot \theta-\theta \csc ^{2} \theta$ and $m(\theta)=-\ell^{\prime}(\theta) / 2=$ $\csc ^{2} \theta(1-\theta \cot \theta)$ we can write 3.23 as

$$
\left.\frac{d}{d c} \lambda_{1}\left(\tilde{H}_{1}(c)\right)\right|_{c=1}=\frac{1}{2}(n-1) \frac{\int_{0}^{\theta_{1}}[(n+1) m(\theta)-(n-1)] v_{1}^{2} d \theta}{\int_{0}^{\theta_{1}} v_{1}^{2} d \theta}
$$

and 3.24 as

$$
\left.\frac{d}{d c} \lambda_{1}\left(\tilde{H}_{0}(c)\right)\right|_{c=1}=\frac{1}{2}(n-1) \frac{\int_{0}^{\theta_{1}}[(n-3) m(\theta)-(n-1)] v_{0}^{2} d \theta}{\int_{0}^{\theta_{1}} v_{0}^{2} d \theta}
$$

respectively. Using the relations $m(\theta)=-\ell^{\prime}(\theta) / 2, \ell(\theta) \cot \theta=m(\theta)-1$, and $v_{0}^{2}=u_{0}^{2} \sin ^{n-1} \theta$, we can rewrite the numerator of the right-hand side of (3.26) as

$$
\begin{aligned}
\int_{0}^{\theta_{1}}[( & n-1)(m(\theta)-1)-2 m(\theta)] v_{0}^{2} d \theta \\
\quad= & \int_{0}^{\theta_{1}}\left[(n-1) \ell(\theta) \cot \theta+\ell^{\prime}(\theta)\right] u_{0}^{2} \sin ^{n-1} \theta d \theta \\
\quad= & \int_{0}^{\theta_{1}}\left[\ell(\theta) \sin ^{n-1} \theta\right]^{\prime} u_{0}^{2} d \theta \\
= & \int_{0}^{\theta_{1}}\left[-2 \ell(\theta) u_{0}(\theta) u_{0}^{\prime}(\theta)\right] \sin ^{n-1} \theta d \theta,
\end{aligned}
$$

where the last equality follows by integrating by parts (both boundary terms vanish). Finally, from (3.26) and (3.27) we obtain

$$
\left.\frac{d}{d c} \lambda_{1}\left(\tilde{H}_{0}(c)\right)\right|_{c=1}=(n-1) \frac{\int_{0}^{\theta_{1}}\left[-\ell(\theta) u_{0}(\theta) u_{0}^{\prime}(\theta)\right] \sin ^{n-1} \theta d \theta}{\int_{0}^{\theta_{1}} v_{0}^{2} d \theta} .
$$

At this point we need the following properties of the functions $\ell(\theta)$ and $m(\theta)$.

Lemma 3.3. The function

$$
\ell(\theta) \equiv \cot \theta-\theta \csc ^{2} \theta
$$

is negative, decreasing, and concave for $0<\theta<\pi$. Moreover, the function

$$
m(\theta) \equiv-\frac{1}{2} \ell^{\prime}(\theta)=\csc ^{2} \theta(1-\theta \cot \theta)
$$

is positive, increasing, and convex for $0<\theta<\pi$. Also, $m(0)=1 / 3, m(\pi / 2)=1$, and $m\left(\pi^{-}\right)=\infty$.

Proof. Using the product representation of $\sin \theta$, i.e., $\sin \theta=\theta \prod_{k=1}^{\infty}\left(1-\theta^{2} /(k \pi)^{2}\right)$, one has

$$
\cot \theta=\sum_{k=-\infty}^{\infty} \frac{1}{\theta+k \pi} \quad \text { and } \quad \csc ^{2} \theta=\sum_{k=-\infty}^{\infty} \frac{1}{(\theta+k \pi)^{2}}
$$


(convergence of the series for $\cot \theta$ here is understood in the sense of symmetric partial sums). From (3.31) we obtain the following representation for $\ell(\theta)$ :

$$
\ell(\theta)=-\sum_{k=1}^{\infty}\left[\frac{k \pi}{(k \pi-\theta)^{2}}-\frac{k \pi}{(k \pi+\theta)^{2}}\right] .
$$

It follows from (3.32) that $\ell(\theta)<0$ for $0<\theta<\pi$. Also from 3.32 we have

$$
m(\theta)=-\frac{\ell^{\prime}(\theta)}{2}=\sum_{k=1}^{\infty}\left[\frac{k \pi}{(k \pi+\theta)^{3}}+\frac{k \pi}{(k \pi-\theta)^{3}}\right],
$$

which is positive for $0<\theta<\pi$. Thus, $m(\theta)$ is positive and $\ell(\theta)$ is decreasing for $0<\theta<\pi$. Taking derivatives again, we find

$$
m^{\prime}(\theta)=3 \sum_{k=1}^{\infty}\left[\frac{k \pi}{(k \pi-\theta)^{4}}-\frac{k \pi}{(k \pi+\theta)^{4}}\right] .
$$

The right-hand side of (3.34) is positive for $\theta \in(0, \pi)$. Hence, $m(\theta)$ is increasing and $\ell(\theta)$ is concave in $(0, \pi)$. It also follows from (3.34) that $m^{\prime}(\theta)$ is increasing, and therefore $m(\theta)$ is convex in $\left(0, \theta_{1}\right)$. Lastly, the values of $m(\theta)$ at $\theta=0, \pi / 2$, and $\pi^{-}$are found by explicit evaluation.

Remark. In fact the function $\ell$ and all its derivatives are negative for $0<\theta<\pi$.

With all these preliminary results in hand we are ready to prove Theorems 1.2 and 1.3 .

Proof of Theorem 1.2. Since $\tilde{\lambda}_{1}(c) \equiv \lambda_{1}\left(\tilde{H}_{0}(c)\right)=c^{2} \lambda_{1}\left(c \theta_{1}\right)$ (see (3.16) above), we just need to prove that

$$
\left.\frac{d}{d c} \tilde{\lambda}_{1}(c)\right|_{c=1}=\frac{1}{\theta_{1}} \frac{d}{d \theta_{1}}\left(\theta_{1}^{2} \lambda_{1}\left(\theta_{1}\right)\right)<0 .
$$

This inequality follows from (3.28) and Lemmas 3.2 and 3.3 Note that it holds for all $\theta_{1} \in(0, \pi)$.

Proof of Theorem 1.3. Since $\lambda_{2}\left(\theta_{1}\right)>\lambda_{1}\left(\theta_{1}\right)$ and $d \tilde{\lambda}_{1} /\left.d c\right|_{c=1}<0$, to prove (3.22) and therefore

$$
\frac{d}{d \theta_{1}}\left(\frac{\lambda_{2}\left(\theta_{1}\right)}{\lambda_{1}\left(\theta_{1}\right)}\right)>0
$$

reduces to showing (since $\tilde{\lambda}_{1} \tilde{\lambda}_{2}^{\prime}-\tilde{\lambda}_{2} \tilde{\lambda}_{1}^{\prime}$ can be grouped as $\left.\tilde{\lambda}_{1}\left(\tilde{\lambda}_{2}^{\prime}-\tilde{\lambda}_{1}^{\prime}\right)-\left(\tilde{\lambda}_{2}-\tilde{\lambda}_{1}\right) \tilde{\lambda}_{1}^{\prime}\right)$

$$
\left.\frac{d \tilde{\lambda}_{2}}{d c}\right|_{c=1}-\left.\frac{d \tilde{\lambda}_{1}}{d c}\right|_{c=1}>0
$$

where $\tilde{\lambda}_{2}(c) \equiv \lambda_{1}\left(\tilde{H}_{1}(c)\right)$. From equations (3.25) and (3.26) this is equivalent to proving

$$
(n+1) \frac{\int_{0}^{\theta_{1}} m(\theta) v_{1}^{2} d \theta}{\int_{0}^{\theta_{1}} v_{1}^{2} d \theta}>(n-3) \frac{\int_{0}^{\theta_{1}} m(\theta) v_{0}^{2} d \theta}{\int_{0}^{\theta_{1}} v_{0}^{2} d \theta},
$$

which is obviously true for $n \leq 3$ since $m(\theta)$ is positive. For $n \geq 4$ it suffices to show

$$
\frac{\int_{0}^{\theta_{1}} m(\theta) v_{1}^{2} d \theta}{\int_{0}^{\theta_{1}} v_{1}^{2} d \theta}>\frac{\int_{0}^{\theta_{1}} m(\theta) v_{0}^{2} d \theta}{\int_{0}^{\theta_{1}} v_{0}^{2} d \theta} .
$$


The fact that $g=v_{1} / v_{0}=y_{2} / y_{1}$ is an increasing function of $\theta$ on $\left[0, \theta_{1}\right]$ for $0<$ $\theta_{1} \leq \pi / 2$ (see Section 4 below) implies that $\hat{v}_{1} \equiv v_{1} /\left(\int_{0}^{\theta_{1}} v_{1}^{2} d \theta\right)^{1 / 2}$ and $\hat{v}_{0} \equiv$ $v_{0} /\left(\int_{0}^{\theta_{1}} v_{0}^{2} d \theta\right)^{1 / 2}$ must have exactly one crossing in $\left(0, \theta_{1}\right)$. Since $m(\theta)$ is positive and increasing in $[0, \pi)$, the desired inequality (3.36) follows by applying Lemma 2.7 of [14], p. 69; this inequality is also known as Bank's inequality [15] (see also [27] or [6], p. 607).

Remark. For $n=2$ we can obtain a stronger version of Theorem 1.3 which holds for all $\theta_{1} \in(0, \pi)$. Since $m(\theta)$ is increasing and $m(0)=1 / 3$ we have $(n+1) m(\theta)-$ $(n-1) \geq(n+1) / 3-(n-1)=2(2-n) / 3=0$ if $n=2$. Thus, from (3.25) we have

$$
\left.\frac{d \tilde{\lambda}_{2}}{d c}\right|_{c=1}>0 \quad \text { if } n=2 \text {. }
$$

This inequality together with (3.35) implies (3.36) (or, even more directly, (3.22) and therefore Theorem 1.3 for $n=2$ and $0<\theta_{1}<\pi$. In fact, this argument shows that for all $\theta_{1} \in(0, \pi) \quad \theta_{1}^{2} \lambda_{1}\left(\theta_{1}\right)$ is decreasing and $\theta_{1}^{2} \lambda_{2}\left(\theta_{1}\right)$ is increasing. These lead immediately to the fact that $\lambda_{2}\left(\theta_{1}\right) / \lambda_{1}\left(\theta_{1}\right)$ and $\theta_{1}^{2}\left[\lambda_{2}\left(\theta_{1}\right)-\lambda_{1}\left(\theta_{1}\right)\right]$ are both increasing for $0<\theta_{1}<\pi$. For $n=3$ a related argument allows us to show that $\theta_{1}^{2}\left[\lambda_{2}\left(\theta_{1}\right)-\lambda_{1}\left(\theta_{1}\right)\right]$ is increasing for $0<\theta_{1}<\pi$, and hence that Theorem 1.3 extends to $0<\theta_{1}<\pi$ in that case as well. For $n \geq 4$ we only have a proof of Theorem 1.3 for $0<\theta_{1} \leq \pi / 2$.

Next we prove two inequalities between the first two Dirichlet eigenvalues of a geodesic ball which are needed in Section 4 .

Theorem 3.1. Let $\lambda_{1}$ and $\lambda_{2}$ be the first two eigenvalues of the Dirichlet Laplacian on a geodesic ball contained in a hemisphere of $\mathbb{S}^{n}$. Denote its (geodesic) radius by $\theta_{1}$. Then

$$
\frac{\lambda_{2}-n}{\lambda_{1}} \geq \frac{n+2}{n} \quad \text { for } 0<\theta_{1} \leq \pi / 2
$$

with equality if and only if $\theta_{1}=\pi / 2$ (i.e., for the hemisphere).

Remarks. (1) If we consider $\pi / 2<\theta_{1}<\pi$, then inequality (3.37) is reversed (and is a strict inequality, i.e., $\left(\lambda_{2}-n\right) / \lambda_{1}<(n+2) / n$ for $\left.\pi / 2<\theta_{1}<\pi\right)$. This inequality is actually quite interesting since it allows us to control the way $\lambda_{2}$ goes to $n$ (from above) as $\theta_{1} \rightarrow \pi^{-}$in terms of $\lambda_{1}$ (since $\lambda_{1}$ goes to 0 as $\theta_{1} \rightarrow \pi^{-}$). The proof of this reversed inequality will not be given, since it follows by making suitable modifications to the proof of Theorem 3.1, as given below.

(2) Theorem [3.1 is the analog for $\mathbb{S}^{n}$ of our Lemma 2.2 of [7] for the Euclidean case. Indeed, the inequality of Lemma 2.2 follows (except that we do not get a strict inequality) if we consider (3.37) in the limit $\theta_{1} \rightarrow 0^{+}$(the "Euclidean limit"). Since $\lambda_{1} \approx \alpha^{2} / \theta_{1}^{2}$ and $\lambda_{2} \approx \beta^{2} / \theta_{1}^{2}$ for $\theta_{1}$ near 0 where $\alpha$ and $\beta$ are the Bessel function zeros $j_{n / 2-1,1}$ and $j_{n / 2,1}$ (see [1] for the notation here), the inequality $\beta^{2} / \alpha^{2} \geq(n+2) / n$ follows.

Proof. The proof is similar to that of Lemma 2.2 of [7]. We assume that $\theta_{1}<\pi / 2$ through most of the proof, returning to the case $\theta_{1}=\pi / 2$ (which can be treated explicitly in terms of elementary functions) only at the end. We shall use a suitable 
trial function (based on $y_{2}$ ) in the Rayleigh quotient for $\lambda_{1}=\lambda_{1}\left(\theta_{1}\right)$ :

$$
\lambda_{1} \leq \frac{\int_{0}^{\theta_{1}}\left(u^{\prime}\right)^{2} \sin ^{n-1} \theta d \theta}{\int_{0}^{\theta_{1}} u^{2} \sin ^{n-1} \theta d \theta}=-\frac{\int_{0}^{\theta_{1}} u\left(\sin ^{n-1} \theta u^{\prime}\right)^{\prime} d \theta}{\int_{0}^{\theta_{1}} u^{2} \sin ^{n-1} \theta d \theta} .
$$

It suffices that the trial function $u$ be real and continuous on $\left[0, \theta_{1}\right]$, have $u(0)$ finite and $u\left(\theta_{1}\right)=0$, and be such that all the integrals occurring above exist as finite real numbers. This includes, in particular, the case $u=y_{2} / \sin \theta$, which we now adopt. With this choice we find

$$
\begin{aligned}
-\left(\sin ^{n-1} \theta u^{\prime}\right)^{\prime} & =-\frac{d}{d \theta}\left[\sin ^{n-1} \theta\left(\frac{y_{2}}{\sin \theta}\right)^{\prime}\right] \\
& =-\sin ^{n-2} \theta y_{2}^{\prime \prime}-(n-3) \sin ^{n-2} \theta \cot \theta y_{2}^{\prime} \\
& +(n-3) \sin ^{n-4} \theta y_{2}-(n-2) \sin ^{n-2} \theta y_{2},
\end{aligned}
$$

and, upon using the differential equation satisfied by $y_{2}$ (i.e., (3.1) with $m=1$ ), we have

$$
-\left(\sin ^{n-1} \theta u^{\prime}\right)^{\prime}=2 \sin ^{n-2} \theta \cot \theta y_{2}^{\prime}+\left(\lambda_{2}-n+2\right) \sin ^{n-2} \theta y_{2}-2 \sin ^{n-4} \theta y_{2} .
$$

That the expression on the right here has a finite limit as $\theta \rightarrow 0^{+}$follows from the fact that $y_{2}(\theta)$ can be taken as $u_{1}(\theta)$ and $u_{1}(\theta)=c_{1} \theta+O\left(\theta^{3}\right)$ as $\theta \rightarrow 0^{+}$(see (3.1) and (3.2)). It now follows from (3.38) and (3.40) (since for $\theta_{1}<\pi / 2, u=y_{2} / \sin \theta$ does not satisfy the equation that $y_{1}$ does, we can write a strict inequality here) that

$$
\begin{aligned}
& \lambda_{1} \int_{0}^{\theta_{1}} u y_{2} \sin ^{n-2} \theta d \theta \\
& \quad<\int_{0}^{\theta_{1}} u\left[2 \cot \theta y_{2}^{\prime}+\left(\lambda_{2}-n+2\right) y_{2}-2 \csc ^{2} \theta y_{2}\right] \sin ^{n-2} \theta d \theta .
\end{aligned}
$$

Since we would like to show that $\lambda_{1}<n\left(\lambda_{2}-n\right) /(n+2)$, it will be enough to show that the right-hand side of (3.41) is less than or equal to

$$
\left[n\left(\lambda_{2}-n\right) /(n+2)\right] \int_{0}^{\theta_{1}} u y_{2} \sin ^{n-2} \theta d \theta
$$

or, equivalently,

$$
\begin{aligned}
(n+2) \int_{0}^{\theta_{1}} u\left[2 \cot \theta y_{2}^{\prime}+\left(\lambda_{2}-n+2\right) y_{2}\right. & \left.-2 \csc ^{2} \theta y_{2}\right] \sin ^{n-2} \theta d \theta \\
& \leq n\left(\lambda_{2}-n\right) \int_{0}^{\theta_{1}} u y_{2} \sin ^{n-2} \theta d \theta
\end{aligned}
$$

or

$$
2 \int_{0}^{\theta_{1}} u\left[(n+2) \cot \theta y_{2}^{\prime}+\left(\lambda_{2}+2\right) y_{2}-(n+2) \csc ^{2} \theta y_{2}\right] \sin ^{n-2} \theta d \theta \leq 0 .
$$

We now rewrite this integral using $y_{2}=u_{1}\left(\theta ; \lambda_{2}\right)$ (where $\lambda_{2}=\lambda_{2}\left(\theta_{1}\right)$ ) and proceed to simplify the integrand using the relations (3.3), 3.6) developed for the $u_{m}$ 's. First, using (3.3) with $m=1$ the expression in square brackets becomes

$$
(n+2) \cot \theta\left[\cot \theta u_{1}-u_{2}\right]+\left(\lambda_{2}+2\right) u_{1}-(n+2) \csc ^{2} \theta u_{1}
$$


or, since $\cot ^{2} \theta-\csc ^{2} \theta=-1$,

$$
-(n+2) \cot \theta u_{2}+\left(\lambda_{2}-n\right) u_{1} \text {. }
$$

Finally, we use the recursion relation (3.6) with $m=2$ to see that

$$
u_{3}=(n+2) \cot \theta u_{2}-(\lambda-n) u_{1}
$$

and hence, since we are taking $\lambda=\lambda_{2}$, inequality 3.42 may be rewritten simply as

$$
-2 \int_{0}^{\theta_{1}} u u_{3} \sin ^{n-2} \theta d \theta \leq 0
$$

which clearly holds if $u_{3}>0$ for $0<\theta<\theta_{1}$ since $u=y_{2} / \sin \theta$ and $y_{2}>0$ on $\left(0, \theta_{1}\right)$. To see that $u_{3}>0$ for $0<\theta<\theta_{1}$ we use the relation (3.7), first for $m=1$ and then for $m=2$. With $m=1$ we have

$$
\sin ^{n} \theta u_{2}(\theta)=\left(\lambda_{2}-n\right) \int_{0}^{\theta}(\sin t)^{n} u_{1}(t) d t
$$

showing that $u_{2}\left(\theta ; \lambda_{2}\right)>0$ for $0<\theta \leq \theta_{1}$ since $u_{1}\left(\theta ; \lambda_{2}\right)=y_{2}(\theta)>0$ on $\left(0, \theta_{1}\right)$ and $\lambda_{2}=\lambda_{2}\left(\theta_{1}\right)>n$ for $0<\theta_{1} \leq \pi / 2$ by domain monotonicity of Dirichlet eigenvalues (and the fact that $\left.\lambda_{2}(\pi / 2)=2(n+1)>n\right)$. This in turn yields $u_{3}>0$ for $0<\theta \leq \theta_{1}$ if $0<\theta_{1}<\pi / 2$ since by (3.7) with $m=2$ we have

$$
\sin ^{n+1} \theta u_{3}(\theta)=\left(\lambda_{2}-2(n+1)\right) \int_{0}^{\theta}(\sin t)^{n+1} u_{2}(t) d t
$$

and we know by domain monotonicity that $\lambda_{2}\left(\theta_{1}\right)>\lambda_{2}(\pi / 2)=2(n+1)$ for $0<$ $\theta_{1}<\pi / 2$.

Thus we have proved inequality (3.37) of our theorem for $0<\theta_{1}<\pi / 2$ (with strict inequality in (3.37)). It only remains to show that equality holds when $\theta_{1}=\pi / 2$ and this is elementary since in this case we can solve explicitly for all eigenvalues and eigenfunctions without even the need of special functions. One finds that $\lambda_{1}=n$ with eigenfunction $x_{n+1}=\cos \theta$ and that $\lambda_{2}=2(n+1)$ with exactly $n$ linearly independent eigenfunctions $x_{i} x_{n+1}$ for $i=1,2, \ldots, n$. Clearly $\left(\lambda_{2}-n\right) / \lambda_{1}=(n+2) / n$ and our proof is complete.

Remark. To fill in the picture for the hemisphere in $\mathbb{S}^{n}$, we note that $y_{1}=\cos \theta$ and $y_{2}=\sin \theta \cos \theta$, and thus $u_{1}=\sin \theta \cos \theta, u_{2}=\sin ^{2} \theta$, and $u_{3} \equiv 0$. In this case $u=y_{2} / \sin \theta \equiv y_{1}$ and (3.38) becomes an equality.

Lemma 3.4. With notation as above,

$$
\lambda_{2}-\lambda_{1}>\frac{n-1}{\sin ^{2} \theta_{1}}
$$

for $0<\theta_{1} \leq \pi / 2$.

Proof. The proof is similar to that of Lemma 2.1 of 7 ]. We use $u=y_{2}$ in the Rayleigh quotient for $\lambda_{1}$ (i.e., in (3.38) above). Since

$$
-\left(\sin ^{n-1} \theta y_{2}^{\prime}\right)^{\prime}=\sin ^{n-1} \theta\left(\lambda_{2}-\frac{n-1}{\sin ^{2} \theta}\right) y_{2},
$$

we get from 3.38

$$
\lambda_{1}<\lambda_{2}-\frac{\int_{0}^{\theta_{1}} u^{2}\left((n-1) / \sin ^{2} \theta\right) \sin ^{n-1} \theta d \theta}{\int_{0}^{\theta_{1}} u^{2} \sin ^{n-1} \theta d \theta}
$$


(again, $y_{2}$ does not satisfy the equation that $y_{1}$ does, so (3.44) is strict). Now the lemma follows by the monotonicity of $\sin \theta$ in $\left(0, \theta_{1}\right)$ for $0<\theta_{1} \leq \pi / 2$.

To conclude this section we present the following results which are needed in Section 4.

Theorem 3.2. Let $p(\theta)=-u_{0}^{\prime}\left(\theta, \lambda_{1}\right) / u_{0}\left(\theta, \lambda_{1}\right)=u_{1}\left(\theta, \lambda_{1}\right) / u_{0}\left(\theta, \lambda_{1}\right)$. Then, $p(\theta)$ is positive, strictly increasing, and strictly convex on $\left(0, \theta_{1}\right)$ for $0<\theta_{1} \leq \pi / 2$. Moreover, $p(0)=0$ and $p(\theta) \rightarrow \infty$ as $\theta \rightarrow \theta_{1}^{-}$.

Proof. The analog of this result in the Euclidean case was proved in Lemma 2.3 of 7]. That $p(0)=0$ and $p(\theta) \rightarrow \infty$ as $\theta \rightarrow \theta_{1}^{-}$follow from the boundary behavior of $u_{0}$. Using the raising and lowering identities (3.3) and (3.4) with $m=0$ and $m=1$ respectively and with $\lambda$ fixed at $\lambda_{1}\left(\theta_{1}\right)$, i.e.,

$$
\begin{aligned}
u_{1} & =-u_{0}^{\prime}, \\
\lambda_{1} u_{0} & =u_{1}^{\prime}+(n-1) \cot \theta u_{1},
\end{aligned}
$$

one obtains

$$
u_{0}^{2} p^{\prime}=\lambda_{1} u_{0}^{2}+u_{1}^{2}-(n-1) \cot \theta u_{0} u_{1} \equiv \sigma(\theta),
$$

and in similar fashion

$$
\left(\sin ^{n-1} \theta \sigma(\theta)\right)^{\prime}=(n-1) \sin ^{n-3} \theta u_{0} u_{1} .
$$

The function $\sigma(\theta)$ is finite at $\theta=0$, and thus $\sigma(\theta) \sin ^{n-1} \theta=0$ at $\theta=0$. Since $u_{0}$ is positive and decreasing in $\left(0, \theta_{1}\right), u_{0} u_{1}=-u_{0} u_{0}^{\prime}>0$ there. Hence, $\sigma(\theta)>0$ in $\left(0, \theta_{1}\right]$ and therefore by (3.46) $p$ is increasing there. Clearly $p(\theta)>0$ in $\left(0, \theta_{1}\right)$, since $p(0)=0$ and $p$ is increasing there. Moreover, from (3.46) we find

$$
u_{0}^{2} p^{\prime \prime}=(n-1) \frac{u_{0}^{2} p}{\sin ^{2} \theta}-(n-1) \cot \theta \sigma+2 p \sigma \equiv s(\theta) .
$$

From (3.45), (3.46), (3.47), and (3.48) we obtain

$$
\left(\sin ^{n+1} \theta s(\theta)\right)^{\prime}=2 \sin ^{n} \theta \sigma(\theta)\left\{\frac{\sigma}{u_{0}^{2}} \sin \theta+(n-1) \sin \theta+2 p \cos \theta\right\}>0
$$

in $\left(0, \theta_{1}\right)$ for $0<\theta_{1} \leq \pi / 2$. Since $\sin ^{n+1} \theta s(\theta)=0$ at $\theta=0$, this implies that $s(\theta)>$ 0 in $\left(0, \theta_{1}\right)$ and therefore, by (3.48), $p^{\prime \prime}>0$ there and the proof is complete.

Remark. The function $p$ satisfies the Riccati equation

$$
p^{\prime}=\lambda_{1}+p^{2}-(n-1) \cot \theta p .
$$

An alternative proof of Theorem 3.2 can be given directly from (3.50). In particular, the fact that $p$ is increasing follows from 3.50 and the convexity of $\cot \theta$ in $(0, \pi / 2)$ using arguments similar to the ones used to prove Theorem 4.1 below.

Lemma 3.5. The function $p(\theta) \cot \theta$ is strictly increasing on $\left(0, \theta_{1}\right)$ for $0<\theta_{1}<$ $\pi / 2$. If $\theta_{1}=\pi / 2, p(\theta)=\tan \theta$, so $p(\theta) \cot \theta \equiv 1$ in that case.

Proof. Consider the function $r(\theta) \equiv p(\theta) \cot \theta-\lambda_{1} / n$. Using (3.47), (3.48), and (3.50) one can show that $r(\theta)$ satisfies the equation

$$
\left(\sin ^{n-1} \theta u_{0}^{2} r^{\prime}\right)^{\prime}=2 n \sin ^{n-3} \theta u_{0}^{2} r .
$$


Since the function $p(\theta)$ is odd and analytic in $\theta$ for $\theta$ near 0 , we can expand it in odd powers of $\theta$. Inserting a series expansion in the Riccati equation (3.50) we find

$$
p(\theta)=\frac{\lambda_{1}}{n} \theta+\frac{\lambda_{1}}{3 n^{2}(n+2)}\left(3 \lambda_{1}+n(n-1)\right) \theta^{3}+O\left(\theta^{5}\right)
$$

for $\theta$ near 0 . Substituting this into the definition of $r(\theta)$ we find

$$
r(\theta) \approx \frac{\lambda_{1}}{n^{2}(n+2)}\left(\lambda_{1}-n\right) \theta^{2}
$$

as $\theta \rightarrow 0^{+}$. Since $\lambda_{1}\left(\theta_{1}\right)>n$ for $\theta_{1}<\pi / 2$ (which follows from the fact that $\lambda_{1}(\pi / 2)=n$ and $\lambda_{1}\left(\theta_{1}\right)$ is decreasing in $\left.\theta_{1}\right), r(\theta)$ is positive in a neighborhood of 0 . Now, $r(\theta)$ is a continuously differentiable function in $\left[0, \theta_{1}\right)$, it is positive in a neighborhood of 0 , and it goes to infinity as $\theta \rightarrow \theta_{1}^{-}$. This implies that either $r(\theta)$ is an increasing function in $\left[0, \theta_{1}\right)$ or that it has a positive local maximum. However, this latter possibility is ruled out by (3.51) (any possible positive critical point of $r(\theta)$ must be a local minimum). Thus, $r(\theta)$ is increasing on $\left(0, \theta_{1}\right)$ if $0<\theta_{1}<\pi / 2$. If $\theta_{1}=\pi / 2, u_{0}=\cos \theta$, hence $u_{0}^{\prime}=-\sin \theta, p(\theta)=\tan \theta$, and finally $r(\theta) \equiv 0$ since $\lambda_{1}=n$.

Remark. In the Euclidean case, the analog of Lemma 3.5 (i.e., Lemma 2.4 of [7]) was an immediate consequence of the convexity of the function analogous to the function $p(\theta)$ used here. For $\mathbb{S}^{n}$, as we have seen, the proof is somewhat more involved.

\section{Monotonicity properties of $g$ And $B$}

In this section we prove the key properties of the functions that occur in our rearrangement procedure in Section 6. These functions are

$$
q(\theta)=\sin \theta \frac{g^{\prime}(\theta)}{g(\theta)},
$$

and

$$
B(\theta)=g^{\prime}(\theta)^{2}+\frac{n-1}{\sin ^{2} \theta} g(\theta)^{2}=\left[q^{2}+(n-1)\right]\left(\frac{g}{\sin \theta}\right)^{2},
$$

where

$$
g(\theta)=\frac{y_{2}(\theta)}{y_{1}(\theta)}=\frac{u_{1}\left(\theta ; \lambda_{2}\left(\theta_{1}\right)\right)}{u_{0}\left(\theta ; \lambda_{1}\left(\theta_{1}\right)\right)} .
$$

Our objectives in this section are to prove that $g(\theta)$ is increasing and $B(\theta)$ is decreasing in $\left[0, \theta_{1}\right]$ for $0<\theta_{1} \leq \pi / 2$. For the hemisphere (i.e., for $\theta_{1}=\pi / 2$ ) we can explicitly compute $g(\theta)=\sin \theta$ and $B(\theta)=(n-1)+\cos ^{2} \theta$ (see the remark following the proof of Theorem 3.1). It is obvious that $g$ is increasing and $B$ is decreasing in this case. Thus, we can assume in the rest of this section that $\theta_{1}<\pi / 2$.

Since $g$ and $\sin \theta$ are positive, that $g$ is increasing will be a simple consequence of showing $q \geq 0$. On the other hand, from (4.1) and (4.2) it follows that

$$
B^{\prime}(\theta)=2\left[q q^{\prime}-(\cos \theta-q)\left(q^{2}+n-1\right) \frac{1}{\sin \theta}\right]\left(\frac{g(\theta)}{\sin \theta}\right)^{2} .
$$


Hence, that $B$ is decreasing will be a consequence of showing that $q^{\prime} \leq 0$ and $0 \leq q \leq \cos \theta$. Thus, in order to prove the desired properties of $g$ and $B$ we only need to show that $0 \leq q(\theta) \leq \cos \theta$ and $q^{\prime}(\theta) \leq 0$ for $0 \leq \theta \leq \theta_{1}$.

The strategy we use to prove these results for $q$ follows the same general method used in [7] and 9]. Since we shall need the boundary behavior of $q$ at the two endpoints $\theta=0$ and $\theta=\theta_{1}$ (this is necessitated by the fact that the coefficients in the right-hand side of the differential equation for $q$, equation (4.7) below, become singular at the two endpoints), we give these now. By Taylor-Frobenius expansion, we find

$$
\begin{gathered}
q(0)=1, \quad q^{\prime}(0)=0, \quad q^{\prime \prime}(0)=2\left(\frac{\lambda_{1}}{n}-\frac{\lambda_{2}}{n+2}-\frac{2-n}{2(n+2)}\right) \\
q\left(\theta_{1}\right)=0, \quad q^{\prime}\left(\theta_{1}\right)=-\frac{1}{3}\left(\lambda_{2}-\lambda_{1}-\frac{n-1}{\sin ^{2} \theta_{1}}\right) \sin \theta_{1} .
\end{gathered}
$$

Throughout this section we will use $\lambda_{1}$ and $\lambda_{2}$ to denote $\lambda_{1}\left(\theta_{1}\right)$ and $\lambda_{2}\left(\theta_{1}\right)$, respectively. From (4.5) and Theorem 3.1 we have that $q^{\prime \prime}(0)<-1$ for $\theta_{1}<\pi / 2$. From (4.6) and Lemma 3.4 we have that $q^{\prime}\left(\theta_{1}\right)<0$. Therefore, $q<\cos \theta$ on an interval just to the right of 0 and $q>0$ on an interval just to the left of $\theta_{1}$.

In order to prove that $q \geq 0, q^{\prime} \leq 0$, and $q \leq \cos \theta$ for $0 \leq \theta \leq \theta_{1}$ we analyze the ordinary differential equation satisfied by $q$. To obtain the differential equation for $q$ first we differentiate (4.1) with respect to $\theta$, and use our choice of $g=y_{2} / y_{1}$. Then we use the equation (3.1), with $m=0$ and $\lambda=\lambda_{1}\left(\theta_{1}\right)$, satisfied by $y_{1}$, and the same equation, but this time with $m=1$ and $\lambda=\lambda_{2}\left(\theta_{1}\right)$, satisfied by $y_{2}$. Thus, we obtain

$$
q^{\prime}=2 p q-(n-2) q \cot \theta-\frac{q^{2}+1-n}{\sin \theta}-\left(\lambda_{2}-\lambda_{1}\right) \sin \theta
$$

Here, the function $p \equiv-y_{1}^{\prime} / y_{1}$ obeys the Riccati equation

$$
p^{\prime}-p^{2}+(n-1) \cot \theta p-\lambda_{1}=0
$$

associated to equation (3.1) with $m=0$ and $\lambda=\lambda_{1}\left(\theta_{1}\right)$ (note that 4.7) is also a Riccati equation). We proved in Section 3 that $p$ is positive, strictly increasing, and strictly convex in $\left(0, \theta_{1}\right)$, for $0<\theta_{1} \leq \pi / 2, p(0)=0$, and $p \rightarrow \infty$ as $\theta \rightarrow \theta_{1}^{-}$ (see Theorem 3.2).

Having derived the equation for $q$, we are ready to prove the necessary facts about $q$. We start by showing $q \geq 0$ in $\left[0, \theta_{1}\right]$, which we prove by contradiction. Assume $q$ is negative somewhere in $\left[0, \theta_{1}\right]$. Since $q(0)=1$ and $q$ is positive to the left of $\theta_{1}$ (and $q$ is continuous) this implies that there are two points $\alpha, \beta$, say, with $0<\alpha<\beta<\theta_{1}$ such that $q(\alpha)=q(\beta)=0$ and $q^{\prime}(\alpha) \leq 0, q^{\prime}(\beta) \geq 0$. At points in $\left(0, \theta_{1}\right)$ where $q=0$ it follows from (4.7) that

$$
q^{\prime}=\frac{n-1}{\sin \theta}-\left(\lambda_{2}-\lambda_{1}\right) \sin \theta .
$$

Since $\lambda_{2}>\lambda_{1}$ and $\sin \theta$ is increasing in $\theta$ for $0<\theta<\pi / 2$, the right-hand side of (4.9) is strictly decreasing in $\theta$, and hence it is not possible to have $\alpha<\beta$ with $q(\alpha)=q(\beta)=0$ and $q^{\prime}(\alpha) \leq 0, q^{\prime}(\beta) \geq 0$. Therefore $q \geq 0$ in $\left[0, \theta_{1}\right]$. 
The proof that $q \leq \cos \theta$ follows the same ideas. Define the function $\psi=\cos \theta-q$. From (4.7) we get

$$
\psi^{\prime}=\left(\lambda_{2}-\lambda_{1}-n\right) \sin \theta+\left(2 p+\frac{\psi}{\sin \theta}\right)(\psi-\cos \theta)-(n-1) \psi \cot \theta .
$$

We have already shown that $\psi(\theta)=\cos \theta-q(\theta)$ is positive in a neighborhood of $\theta=0$. Also, if $\theta_{1}<\pi / 2, \psi\left(\theta_{1}\right)=\cos \theta_{1}>0$. Now assume that $\psi$ is negative somewhere in $\left[0, \theta_{1}\right]$. This implies that there are two points $r, s$, say, with $0<r<$ $s<\theta_{1}$ such that $\psi(r)=\psi(s)=0$ and $\psi^{\prime}(r) \leq 0, \psi^{\prime}(s) \geq 0$. At points in $\left(0, \theta_{1}\right)$ where $\psi=0$ we have from (4.10) that

$$
\frac{1}{\sin \theta} \psi^{\prime}=\left(\lambda_{2}-\lambda_{1}-n\right)-2 p \cot \theta \text {. }
$$

From Lemma 3.5 it follows that the right-hand side of (4.11) is strictly decreasing in $\theta$, hence it is not possible to have $r<s$ with $\psi(r)=\psi(s)=0$ and $\psi^{\prime}(r) \leq 0$, $\psi^{\prime}(s) \geq 0$. Thus $\psi \geq 0$, and hence $q \leq \cos \theta$ in $\left[0, \theta_{1}\right]$.

Finally, we show that $q(\theta)$ is decreasing in $\left[0, \theta_{1}\right]$. It is convenient now to write (4.7) in an alternative form and use convexity arguments. Specifically we consider

$$
\begin{array}{rl}
q^{\prime}=2 & p(\theta) q+(n-2)(1-q) \cot \theta \\
& +\frac{1-q^{2}}{\sin \theta}+(n-2) \tan (\theta / 2)-\left(\lambda_{2}-\lambda_{1}\right) \sin \theta
\end{array}
$$

and, since $\lambda_{2}-\lambda_{1}>0, q \leq 1$, and $n \geq 2$, the right-hand side of (4.12), $F(\theta, q)$, say, is convex in $\theta$ for fixed $q$ because each of the functions $\cot \theta, \csc \theta, \tan (\theta / 2)$, and $-\sin \theta$ is individually convex on the interval $\left[0, \theta_{1}\right]$ for $0<\theta_{1} \leq \pi / 2$. Also the function $p(\theta)$ is convex on the same interval (see Theorem 3.2 above). To see how these facts imply that $q^{\prime} \leq 0$ observe that if not we could find three points $\alpha_{1}, \alpha_{2}$, $\alpha_{3}$ in $\left(0, \theta_{1}\right)$, where $q\left(\alpha_{1}\right)=q\left(\alpha_{2}\right)=q\left(\alpha_{3}\right), q^{\prime}\left(\alpha_{1}\right)<0, q^{\prime}\left(\alpha_{2}\right)>0$, and $q^{\prime}\left(\alpha_{3}\right)<0$. But then we would have (we use $q$ for the common value of $q\left(\alpha_{i}\right)$ here)

$$
\begin{aligned}
0<q^{\prime}\left(\alpha_{2}\right) & =F\left(\alpha_{2}, q\right)=F\left(\mu \alpha_{1}+(1-\mu) \alpha_{3}, q\right) \\
& <\mu F\left(\alpha_{1}, q\right)+(1-\mu) F\left(\alpha_{3}, q\right)=\mu q^{\prime}\left(\alpha_{1}\right)+(1-\mu) q^{\prime}\left(\alpha_{3}\right)<0,
\end{aligned}
$$

a contradiction. We have used the strict convexity of $F(\theta, q)$ in $\theta$ here. The parameter $\mu$ is strictly between 0 and 1 and determines $\alpha_{2}$ as a convex combination of $\alpha_{1}$ and $\alpha_{3}$, that is, $\alpha_{2}=\mu \alpha_{1}+(1-\mu) \alpha_{3}$. To summarize this section we state the results above as a theorem.

Theorem 4.1. With $q(\theta), B(\theta)$, and $g(\theta)$ as defined in (4.1), (4.2), and (4.3), respectively, the inequalities $0 \leq q \leq \cos \theta$ and $q^{\prime} \leq 0$ hold on $\left[0, \theta_{1}\right]$ for $0<\theta_{1} \leq$ $\pi / 2$. It follows that $g(\theta)$ is increasing and $B(\theta)$ is decreasing there as well.

\section{Chiti's COMPARISON ARGUMENT IN $\mathbb{S}^{n}$}

Here we need an extension of Chiti's comparison result [24, [25], [26], 27] (see also Appendix $\mathrm{A}$ of [6]), given originally for domains in $\mathbb{R}^{n}$, to the case of domains in $\mathbb{S}^{n}$

We let $\mathbb{S}^{n}(\rho)$ denote the $n$-dimensional sphere of radius $\rho$ (hence of constant sectional curvature $\left.\kappa=1 / \rho^{2}\right)$. $\mathbb{S}^{n}$ will always denote $\mathbb{S}^{n}(1)$. Define the function

$$
S_{\rho}(r)=\rho \sin (r / \rho)
$$


It is well known that, in geodesic polar coordinates, the metric on $\mathbb{S}^{n}(\rho)$ is

$$
d s^{2}=d r^{2}+S_{\rho}(r)^{2}|d \omega|^{2},
$$

where $r$ represents geodesic distance from a point and $|d \omega|^{2}$ is the canonical metric for $\mathbb{S}^{n-1}$. One should think of $r$ as $\rho$ times the angle $\theta$ from the north pole. Thus $r$ runs from 0 to $\rho \pi$.

We will always assume that $r=0$ (the north pole) is the center for our spherical rearrangements (this is not a restriction since the metric is identical in geodesic polar coordinates about any point). Then for a bounded domain $\Omega \subset \mathbb{S}^{n}(\rho) \Omega^{\star}$, the spherical rearrangement of $\Omega$, will denote the geodesic ball about $r=0$ having the same volume as $\Omega$, i.e., $\left|\Omega^{\star}\right|=|\Omega|$.

For a nonnegative function $f$ defined on $\Omega$ we define two rearranged functions $f^{\#}$ and $f^{\star}$. The decreasing rearrangement $f^{\#}$ of $f$ is a function from $[0,|\Omega|]$ to $\mathbb{R}$ which is equimeasurable with $f$ and nonincreasing. We will use $s$ as the argument of $f^{\#}$ in most instances. The symmetric decreasing (or spherical decreasing) rearrangement $f^{\star}$ of $f$ is a function defined on $\Omega^{\star}$ which is invariant under rotations about $r=0$, equimeasurable with $f$, and nonincreasing with respect to $r$. $f^{\star}$ is a function of $x \in \Omega^{\star}$ but because of its symmetry we will abuse notation and write $f^{\star}(r)$ where $r$ is the geodesic distance from the center of $\Omega^{\star}$. With this understanding we have

$$
f^{\star}(r)=f^{\#}(A(r))
$$

where

$$
A(r) \equiv s=n C_{n} \int_{0}^{r} S_{\rho}(\tau)^{n-1} d \tau
$$

is the $n$-volume of the geodesic ball of radius $r$ in $\mathbb{S}^{n}(\rho)$. Here $C_{n}=\pi^{n / 2} / \Gamma\left(\frac{n}{2}+1\right)$ denotes the volume of the unit ball in $\mathbb{R}^{n}$ (and $n C_{n}$ is its "surface area", i.e., $\left.\left|\mathbb{S}^{n-1}\right|\right)$. We shall also have occasion to use increasing rearrangements. These will be denoted $f_{\#}$ (the increasing rearrangement of $f$ ) and $f_{\star}$ (the symmetric or spherical increasing rearrangement of $f$ ) and their definitions are analogous to those of $f^{\#}$ and $f^{\star}$, respectively. For further information on rearrangements the reader is referred to the book of Hardy, Littlewood, and Pólya [32] and the many other references given in [6], [51].

Finally, we also need the classical isoperimetric inequality extended to $\mathbb{S}^{n}(\rho)$ in its sharp form. We let $L(t)$ be the function giving the $(n-1)$-dimensional volume of the geodesic ball of radius $r$, i.e.,

$$
L(r)=n C_{n} S_{\rho}(r)^{n-1}=A^{\prime}(r) .
$$

Then, for example, for any domain in $\mathbb{S}^{2}$ one has

$$
L^{2} \geq 4 \pi A-\left(\frac{A}{\rho}\right)^{2}=4 \pi A-\kappa A^{2}
$$

(see, e.g., [41]). Here $A=|\Omega|$ and $L$ is the length of the boundary of $\Omega$. Equality occurs in (5.3) if and only if $\Omega$ is a geodesic ball. In $\mathbb{S}^{n}, n>2$, the sharp classical isoperimetric inequality cannot be given in as explicit a form as (5.3). For a bounded domain $\Omega$ we define $H_{n-1}(\partial \Omega)$ to be the $(n-1)$-dimensional volume of $\partial \Omega$. The isoperimetric inequality on $\mathbb{S}^{n}(\rho)$ then reads

$$
H_{n-1}(\partial \Omega) \geq H_{n-1}\left(\partial \Omega^{\star}\right)
$$


with equality if and only if $\Omega$ is a geodesic ball (see Burago and Zalgaller [21], p. 86, Theorem 10.2.1). In terms of the function $L(r)$ defined above (5.4) may be written

$$
H_{n-1}(\partial \Omega) \geq L(\theta(|\Omega|))=n C_{n} S_{\rho}(\theta(|\Omega|))^{n-1},
$$

where $\theta(s)$ is the inverse function to the function $A$ defined in (5.1).

With all these ingredients we state our extension of Chiti's comparison result (throughout the rest of this section we set $\rho=1$, the extension to arbitrary $\rho$ being straightforward).

Theorem 5.1. Let $\Omega$ be a bounded domain in $\mathbb{S}^{n}$ and let $\lambda_{1}$ and $u_{1}$ denote the first Dirichlet eigenvalue and eigenfunction of the Laplacian on $\Omega$. Let $B_{\lambda_{1}}$ be the geodesic ball of such a radius that $\lambda_{1}$ is also the first Dirichlet eigenvalue of the Laplacian on $B_{\lambda_{1}}$. Let $v_{1}>0$ be the first Dirichlet eigenfunction on $B_{\lambda_{1}}$ and fix its normalization so that $\int_{\Omega} u_{1}^{2} d \sigma=\int_{B_{\lambda_{1}}} v_{1}^{2} d \sigma$.

Then there is a value $r_{1} \in\left(0, \theta\left(\left|B_{\lambda_{1}}\right|\right)\right)$ such that

$$
v_{1}(r) \geq u_{1}^{\star}(r) \quad \text { for } r \in\left[0, r_{1}\right],
$$

and

$$
v_{1}(r) \leq u_{1}^{\star}(r) \quad \text { for } r \in\left[r_{1}, \theta\left(\left|B_{\lambda_{1}}\right|\right)\right],
$$

where $\theta(s)$ is the function defined following (5.5) above.

Proof. Let $u_{1}$, respectively $\lambda_{1}$, be the lowest eigenfunction, respectively eigenvalue, of the Dirichlet problem on $\Omega \subset \mathbb{S}^{n}$, i.e.,

$$
-\Delta u_{1}=\lambda_{1} u_{1} \quad \text { in } \Omega, \quad u_{1}=0 \quad \text { on } \partial \Omega .
$$

Define $\Omega_{t}=\left\{x \mid u_{1}(x)>t\right\}$ and $\partial \Omega_{t}=\left\{x \mid u_{1}(x)=t\right\}$. Let $\mu_{1}(t)=\left|\Omega_{t}\right|$, and $\left|\partial \Omega_{t}\right| \equiv H_{n-1}\left(\partial \Omega_{t}\right)$, where $H_{n-1}(d \sigma)$ denotes $(n-1)$-dimensional measure on $\mathbb{S}^{n}$. Then we have (see, e.g., Talenti [51], p. 709, eq. (32))

$$
-\mu_{1}^{\prime}(t)=\int_{\Omega_{t}} \frac{1}{\left|\nabla u_{1}\right|} H_{n-1}(d \sigma),
$$

for almost every $t>0$. Applying Gauss's theorem to (5.8), we have

$$
\lambda_{1} \int_{\Omega_{t}} u_{1} d \sigma=\int_{\partial \Omega_{t}}\left|\nabla u_{1}\right| H_{n-1}(d \sigma),
$$

since the outward normal to $\Omega_{t}$ is given by $-\nabla u_{1} /\left|\nabla u_{1}\right|$. Using the Cauchy-Schwarz inequality and equations (5.9) and (5.10) we obtain

$$
\left|\partial \Omega_{t}\right|^{2}=\left(\int_{\partial \Omega_{t}} H_{n-1}(d \sigma)\right)^{2} \leq\left(-\mu_{1}^{\prime}(t)\right) \lambda_{1} \int_{\Omega_{t}} u_{1} d \sigma .
$$

As discussed above, if $\Omega$ is a domain in $\mathbb{S}^{n}$, the classical isoperimetric inequality is given by

$$
H_{n-1}(\partial \Omega) \geq H_{n-1}\left(\partial \Omega^{\star}\right)
$$

where $\Omega^{\star}$ is a geodesic ball having the same volume as $\Omega$. The $(n-1)$-dimensional measure of $\partial \Omega^{\star}, H_{n-1}\left(\partial \Omega^{\star}\right)$, is given in terms of $\theta_{1}\left(\Omega^{\star}\right)$, the geodesic radius of $\Omega^{\star}$, by

$$
H_{n-1}\left(\partial \Omega^{\star}\right)=n C_{n}\left(\sin \theta_{1}\left(\Omega^{\star}\right)\right)^{n-1},
$$


where $C_{n}=\pi^{n / 2} / \Gamma\left(\frac{n}{2}+1\right)$ is the volume of the unit ball in $\mathbb{R}^{n}$ (and $n C_{n}$ is the volume of $\left.\mathbb{S}^{n-1}\right)$. Therefore, from (5.12) and (5.13) it follows that

$$
\left|\partial \Omega_{t}\right|=H_{n-1}\left(\partial \Omega_{t}\right) \geq n C_{n}\left(\sin \theta_{1}\left(\Omega_{t}^{\star}\right)\right)^{n-1} .
$$

Hence, from (5.11) we have

$$
\lambda_{1} \int_{\Omega_{t}} u_{1} d \sigma \geq n^{2} C_{n}^{2}\left[\sin \theta_{1}\left(\Omega_{t}^{\star}\right)\right]^{2 n-2}\left(-\frac{1}{\mu_{1}^{\prime}(t)}\right) .
$$

Finally one uses the fact that

$$
\int_{\Omega_{t}} u_{1} d \sigma=\int_{0}^{\mu_{1}(t)} u_{1}^{\#}(s) d s
$$

which follows directly from the definition of $u_{1}^{\#}$, the decreasing rearrangement of $u_{1}$ on the interval $[0,|\Omega|]$ (here $s$ is a variable denoting volume and is related to the geodesic radial variable $\theta$ via $s=n C_{n} \int_{0}^{\theta}(\sin r)^{n-1} d r$, i.e., $\left.d s / d \theta=n C_{n}(\sin \theta)^{n-1}\right)$.

Since $u_{1}^{\#}(s)$ is the inverse function to $\mu_{1}(t)$, we have

$$
-\frac{d u_{1}^{\#}}{d s}=-\frac{1}{\mu_{1}^{\prime}(t)}
$$

which, combined with (5.15) and (5.16), yields

$$
-\frac{d u_{1}^{\#}}{d s} \leq \lambda_{1} n^{-2} C_{n}^{-2}(\sin \theta(s))^{2-2 n} \int_{0}^{s} u_{1}^{\#}\left(s^{\prime}\right) d s^{\prime} .
$$

Now, if we view $v_{1}$ as a function of the volume $s$ (here we will abuse notation and continue to call it $v_{1}$ ) rather than as a function of $\theta$ (or $r$ ), with $s=$ $n C_{n} \int_{0}^{\theta}(\sin r)^{n-1} d r$, it satisfies (5.17) with equality, i.e.,

$$
-\frac{d v_{1}}{d s}=\lambda_{1} n^{-2} C_{n}^{-2}(\sin \theta(s))^{2-2 n} \int_{0}^{s} v_{1}\left(s^{\prime}\right) d s^{\prime} .
$$

In fact, (5.18) is an integrated version of equation (3.1) with $m=0$ and $\lambda=\lambda_{1}$ in the variable $s$. Having obtained the relations (5.17) and (5.18) for $u_{1}^{\#}(s)$ and $v_{1}(s)$ respectively, we will prove that under the normalization condition imposed on them, they are either identical or they cross only once in the interval $\left(0,\left|B_{\lambda_{1}}\right|\right)$ (in the sense specified by Theorem 5.1). All the arguments we give below depend on the continuity of $u_{1}^{\#}$ and $v_{1}$. The function $v_{1}$ is in fact real analytic in $\left[0,\left|B_{\lambda_{1}}\right|\right]$ and, furthermore, it is decreasing there as can be seen from Lemma 3.2 above (or from (5.18) and the fact that $\left.v_{1}>0\right)$. The absolute continuity of $u_{1}^{\#}$ on $[0,|\Omega|]$ follows from arguments in [51]. Since $u_{1}^{\#}$ and $v_{1}$ are normalized to have the same $L^{2}-$ norm, they either are identical or they cross. If they coincide, then $B_{\lambda_{1}}=\Omega^{\star}$, and Theorem 5.1 is proved since any $r \in\left(0, \theta\left(\left|B_{\lambda_{1}}\right|\right)\right)$ will serve as $r_{1}$. Next, following Chiti 26] we conclude that $u_{1}^{\#}(0)$ cannot exceed $v_{1}(0)$. In fact, if $u_{1}^{\#}(0) \geq v_{1}(0)$ it follows by mimicking the proof of the main theorem in [26] that $v_{1}(s) \leq u_{1}^{\#}(s)$ for all $s \in\left[0,\left|B_{\lambda_{1}}\right|\right)$ which, in turn, implies $v_{1}(s) \equiv u_{1}^{\#}(s)$ and we are back in the previous case. Thus, if $v_{1}(s) \not \equiv u_{1}^{\#}(s), v_{1}(s)>u_{1}^{\#}(s)$ in a neighborhood of 0 , and both functions being of the same norm they must cross at least once. Let $s_{1}$ be the largest $s \in\left(0,\left|B_{\lambda_{1}}\right|\right)$ such that $u_{1}^{\#}\left(s^{\prime}\right) \leq v_{1}\left(s^{\prime}\right)$ for all $s^{\prime} \leq s$. By the definition 
of $s_{1}$, there is an interval immediately to the right of $s_{1}$ on which $u_{1}^{\#}(s)>v_{1}(s)$. Indeed, by continuity and the definition of $s_{1}$

$$
\int_{0}^{s}\left[u_{1}^{\#}\left(s^{\prime}\right)-v_{1}\left(s^{\prime}\right)\right] d s^{\prime}<0 \quad \text { for } 0<s \leq s_{1}+\epsilon
$$

for some $\epsilon>0$. It now follows that $u_{1}^{\#}(s)>v_{1}(s)$ at least on the interval from $s_{1}$ to $s_{1}+\epsilon$ since by the absolute continuity of $u_{1}^{\#}$

$$
\begin{aligned}
v_{1}(s) & -u_{1}^{\#}(s)=\int_{s_{1}}^{s}\left[\frac{d}{d s}\left(v_{1}-u_{1}^{\#}\right)\right] d s \\
& \leq \lambda_{1} n^{-2} C_{n}^{-2} \int_{s_{1}}^{s}\left(\sin \theta\left(s^{\prime}\right)\right)^{2-2 n} \int_{0}^{s^{\prime}}\left[u_{1}^{\#}\left(s^{\prime \prime}\right)-v_{1}\left(s^{\prime \prime}\right)\right] d s^{\prime \prime} d s^{\prime} \\
& <0 \quad \text { for } s \in\left(s_{1}, s_{1}+\epsilon\right]
\end{aligned}
$$

by virtue of (5.19).

We will now show that $u_{1}^{\#}(s)>v_{1}(s)$ for all $s \in\left(s_{1},\left|B_{\lambda_{1}}\right|\right]$, which will prove the theorem. If not, then the point $s_{2}$ defined to be the largest $s \in\left(s_{1},\left|B_{\lambda_{1}}\right|\right]$ for which $u_{1}^{\#}\left(s^{\prime}\right)>v_{1}\left(s^{\prime}\right)$ for all $s_{1}<s^{\prime}<s$, would be less than $\left|B_{\lambda_{1}}\right|$, and we would have

$$
u_{1}^{\#}(s)>v_{1}(s) \quad \text { for } s \in\left(s_{1}, s_{2}\right)
$$

with $u_{1}^{\#}\left(s_{1}\right)=v_{1}\left(s_{1}\right)$ and $u_{1}^{\#}\left(s_{2}\right)=v_{1}\left(s_{2}\right)$. In this case, we can define the function

$$
w(s)= \begin{cases}v_{1}(s) & \text { for } s \in\left[0, s_{1}\right] \cup\left[s_{2},\left|B_{\lambda_{1}}\right|\right], \\ u_{1}^{\#}(s) & \text { for } s \in\left(s_{1}, s_{2}\right) .\end{cases}
$$

It follows from (5.17) and (5.18) that $w$ satisfies

$$
-\frac{d w}{d s}(s) \leq \lambda_{1} n^{-2} C_{n}^{-2}(\sin \theta(s))^{2-2 n} \int_{0}^{s} w\left(s^{\prime}\right) d s^{\prime},
$$

with strict inequality for all $s>s_{1}$. From $w(s)$ define the function

$$
g(x)=w(s(\theta))
$$

for $x \in B_{\lambda_{1}}$ where $\theta$ is the polar angle (angle from the north pole) corresponding to $x$. Thus $g$ is a radial function on $B_{\lambda_{1}}$ (assumed centered at the norh pole). Because of (5.22) (or (5.20) or (5.21) $), g$ cannot be the groundstate of the Laplacian with Dirichlet boundary conditions on $B_{\lambda_{1}}$ (but it is certainly an admissible trial function for $\left.\lambda_{1}\right)$. Therefore,

$$
\lambda_{1}<\frac{\int_{B_{\lambda_{1}}}|\nabla g|^{2} d \sigma}{\int_{B_{\lambda_{1}}} g^{2} d \sigma} .
$$

By a standard change of variables,

$$
\int_{B_{\lambda_{1}}} g^{2} d \sigma=\int_{0}^{\left|B_{\lambda_{1}}\right|} w^{2}(s) d s
$$

and

$$
\int_{B_{\lambda_{1}}}|\nabla g|^{2} d \sigma=n^{2} C_{n}^{2} \int_{0}^{\left|B_{\lambda_{1}}\right|}(\sin \theta(s))^{2 n-2} w^{\prime}(s)^{2} d s .
$$


Using (15.22) (substitute for one of the $w^{\prime}(s)$ 's in (5.25), using the fact that $-w^{\prime}(s)>$ 0 ) and integration by parts we get

$$
\int_{B_{\lambda_{1}}}|\nabla g|^{2} d \sigma \leq \lambda_{1} \int_{0}^{\left|B_{\lambda_{1}}\right|} w(s)^{2} d s .
$$

From (5.23), (5.24), and (5.26) we get a contradiction, and the theorem follows.

\section{The MAIN Result}

After all the preliminaries developed in Sections 2 through 5 we are ready to prove our main result, i.e., Theorem 1.1 from which the PPW result for domains contained in a hemisphere of $\mathbb{S}^{n}$ follows as indicated in the introduction. As in our proof of the PPW conjecture for domains in $\mathbb{R}^{n}$, the starting point here is the use of the gap inequality, which is a variational estimate for the difference between the first two eigenvalues of the Laplacian. The gap inequality states that

$$
\lambda_{2}(\Omega)-\lambda_{1}(\Omega) \leq \frac{\int_{\Omega}|\nabla P|^{2} u_{1}^{2} d \sigma}{\int_{\Omega} P^{2} u_{1}^{2} d \sigma}
$$

provided $\int_{\Omega} P u_{1}^{2} d \sigma=0$ and $P \not \equiv 0$. Here $\Omega$ is a domain in $\mathbb{S}^{n}$ and $d \sigma$ is the standard volume element in $\mathbb{S}^{n}$. The gap inequality follows from the Rayleigh-Ritz inequality for $\lambda_{2}$ using $P u_{1}$ as the trial function (hence the side condition $P u_{1} \perp u_{1}$ ) after a suitable integration by parts. To get the desired isoperimetric result out of this, one must make very special choices of the function $P$; in particular, choices such that (6.1) is an equality if $\Omega$ is the appropriate geodesic ball.

A key element needed to guarantee the orthogonality of the trial functions $P_{i} u_{1}$ to $u_{1}$ which we will use in the sequel is the center of mass argument embodied in Theorem 2.1 above.

Concerning the choice of trial functions $P_{i}$ we proceed as follows. Thinking of $\mathbb{S}^{n}$ as the unit sphere in $\mathbb{R}^{n+1}$ and with the center of mass point for $\Omega$ fixed at the north pole we take

$$
P_{i}=g(\theta) \frac{x_{i}}{\sin \theta}, \quad i=1,2, \ldots, n,
$$

where $\theta$ represents the angle of a point from the positive $x_{n+1}$-axis (the direction of the north pole). In $\mathbb{S}^{n}$ the variable $\theta$ is the geodesic radial variable with respect to the north pole. Division by $\sin \theta=\sqrt{1-x_{n+1}^{2}}$ normalizes the $n$-vector $\left(x_{1}, x_{2}, \ldots, x_{n}\right)$. The choice of $g(\theta)$, as in the Euclidean case, is determined by the fact that we must have equality in (6.1) when $\Omega$ is a geodesic ball. For a geodesic ball of radius $\theta_{1}, u_{1}=c_{1} y_{1}$ where $y_{1}$ satisfies

$$
y_{1}^{\prime \prime}+(n-1) \cot \theta y_{1}^{\prime}+\lambda_{1} y_{1}=0
$$

with boundary conditions $y_{1}(0)$ finite and $y_{1}\left(\theta_{1}\right)=0$. This is just equation (3.1) with $m=0$ and $\lambda=\lambda_{1}$. Also, $u_{2}=c_{2}\left(x_{i} / \sin \theta\right) y_{2}$ (for any $i=1,2, \ldots, n$ ) where $y_{2}$ satisfies

$$
y_{2}^{\prime \prime}+(n-1) \cot \theta y_{2}^{\prime}+\left(\lambda_{2}-\frac{n-1}{\sin ^{2} \theta}\right) y_{2}=0
$$

with boundary conditions $y_{2}(0)=y_{2}\left(\theta_{1}\right)=0$ (this is just equation (3.1) with $m=1$ and $\lambda=\lambda_{2}$ ). The values $\lambda_{1}$ and $\lambda_{2}$ are to be taken as the least eigenvalues of these one-dimensional radial problems. By Lemma[3.1 these are the correct identifications 
of $\lambda_{1}$ and $\lambda_{2}$ for our geodesic ball. One can express $y_{1}$ and $y_{2}$ in terms of associated Legendre functions. In fact,

$$
y_{1}(\theta)=(\sin \theta)^{1-n / 2} P_{\nu_{1}}^{-(n / 2-1)}(\cos \theta)
$$

and

$$
y_{2}(\theta)=(\sin \theta)^{1-n / 2} P_{\nu_{2}}^{-n / 2}(\cos \theta)
$$

up to unimportant constant factors, where the parameters $\nu_{1}$ and $\nu_{2}$ are related to the eigenvalues $\lambda_{1}$ and $\lambda_{2}$ respectively by

$$
\nu(\nu+1)=\lambda+\frac{n(n-2)}{4} .
$$

We follow Abramowitz and Stegun [1] in our notation here; note that their convention for associated Legendre functions makes $(\sin \theta)^{1-n / 2} P_{\nu}^{-\mu}$, where $\mu=n / 2-$ $1+m$ and $m$ is a nonnegative integer, and not $(\sin \theta)^{1-n / 2} P_{\nu}^{\mu}$, the "right" $n$ dimensional generalization of the familiar associated Legendre functions $P_{\nu}^{m}, m=$ $0,1, \ldots$, from $\mathbb{S}^{2}$ (or $\mathbb{R}^{3}$ ). This means, in particular, that when $n$ is even $P_{\nu}^{\mu}$ can be substituted for $P_{\nu}^{-\mu}$ (they are then proportional), while if $n$ is odd $Q_{\nu}^{\mu}$ can be used (the distinction here is between $\mu$ being an integer or half an odd integer). Using $P_{\nu}^{-\mu}$ with $\mu$ as above circumvents this "even-odd effect". Since we want equality in (6.1) when $\Omega$ is a geodesic ball, using the form of $u_{1}$ and $u_{2}$ for a geodesic ball we see that $g(\theta)$ must be essentially the quotient of $y_{2}$ by $y_{1}$.

Let $\Omega \subset \mathbb{S}^{n}$ be contained in a hemisphere. Let $B_{\lambda_{1}}$ denote the geodesic ball in $\mathbb{S}^{n}$ having the same value of $\lambda_{1}$ as $\Omega$. By Sperner's inequality 49] (see also 31]) $\lambda_{1}\left(B_{\lambda_{1}}\right)=\lambda_{1}(\Omega) \geq \lambda_{1}\left(\Omega^{\star}\right)$ and by the properties of $\lambda_{1}$ for geodesic balls (in particular, $\lambda_{1}$ decreases as the radius of the ball increases), we see that $\theta_{1} \leq \pi / 2$, where $\theta_{1}$ denotes the geodesic radius of $B_{\lambda_{1}}$. We now set $g(\theta)=y_{2}(\theta) / y_{1}(\theta)$ for $0 \leq \theta \leq \theta_{1}, g(\theta)=g\left(\theta_{1}\right)$ for $\theta_{1} \leq \theta \leq \pi / 2$ and we extend $g(\theta)$ to a function on $[0, \pi]$ by reflection about $\theta=\pi / 2$. (Note that this definition makes $B(\theta)=g^{\prime}(\theta)^{2}+$ $(n-1) g(\theta)^{2} / \sin ^{2} \theta$ a decreasing function on $\left[\theta_{1}, \pi / 2\right]$, since $B(\theta)=(n-1) g\left(\theta_{1}\right)^{2} /$ $\sin ^{2} \theta$ there.) We then apply the center of mass result (Theorem 2.1) to $\Omega$ and $\tilde{G}(\theta)=g(\theta) / \sin \theta$ obtaining a choice of Cartesian coordinate axes such that

$$
\int_{\Omega} g(\theta) \frac{x_{i}}{\sin \theta} u_{1}^{2} d \sigma=0, \quad \text { for } i=1,2, \ldots, n .
$$

Using the functions $P_{i}=g(\theta) x_{i} / \sin \theta$ in (6.1) we get

$$
\left(\lambda_{2}-\lambda_{1}\right) \int_{\Omega} g(\theta)^{2}\left(\frac{x_{i}}{\sin \theta}\right)^{2} u_{1}^{2} d \sigma \leq \int_{\Omega}\left|\nabla\left(g(\theta) \frac{x_{i}}{\sin \theta}\right)\right|^{2} u_{1}^{2} d \sigma
$$

for $1 \leq i \leq n$, and summing on $i$ from 1 to $n$ we obtain

$$
\lambda_{2}-\lambda_{1} \leq \frac{\int_{\Omega} B(\theta) u_{1}^{2} d \sigma}{\int_{\Omega} g(\theta)^{2} u_{1}^{2} d \sigma},
$$

where

$$
B(\theta)=g^{\prime}(\theta)^{2}+\frac{n-1}{\sin ^{2} \theta} g(\theta)^{2}
$$

as defined previously (see equation (4.2)). We observe that, in spite of the fact that $\Omega$ is contained in a hemisphere, our use of the center of mass result may imply that $\Omega$ does not lie in the northern hemisphere (i.e., $\theta$ would not be in $(0, \pi / 2)$ for all points in $\Omega$ ). To remedy this situation, we observe that since $\Omega$ is contained 
in a hemisphere, $-\Omega \subset \mathbb{S}^{n} \backslash \Omega$. Thus, if we let $\Omega_{ \pm}=\left\{\vec{x} \in \Omega \mid \pm x_{n+1}>0\right\}$ we have $\Omega_{+} \cap\left(-\Omega_{-}\right)=\emptyset$. Since $g(\theta)$ and $B(\theta)$ are both symmetric with respect to $\theta=\pi / 2$ it follows that the integrals over $\Omega$ can be replaced by integrals over $\tilde{\Omega}=\Omega_{+} \cup\left(-\Omega_{-}\right)$with no change in their values if we agree to transplant $u_{1}$ along with $\Omega_{-}$to $-\Omega_{-}$. This follows since $g$ and $B$ were defined to be symmetric about $\theta=\pi / 2$ and therefore they transplant into themselves. After moving the whole problem to the northern hemisphere (where $g$ is increasing and $B$ is decreasing) we can carry out all the further rearrangements in exact parallel with the Euclidean case, encountering no further difficulties.

To conclude the proof of Theorem 1.1 we need the following two chains of inequalities. We have

$$
\begin{aligned}
\int_{\Omega} B(\theta) u_{1}^{2} d \sigma=\int_{\tilde{\Omega}} B(\theta) \tilde{u}_{1}^{2} d \sigma & \leq \int_{\Omega^{\star}} B(\theta)^{\star} u_{1}^{\star 2} d \sigma \\
& \leq \int_{\Omega^{\star}} B(\theta) u_{1}^{\star 2} d \sigma \leq \int_{B_{\lambda_{1}}} B(\theta) v_{1}^{2} d \sigma
\end{aligned}
$$

and

$$
\begin{aligned}
\int_{\Omega} g(\theta)^{2} u_{1}^{2} d \sigma=\int_{\tilde{\Omega}} g(\theta)^{2} \tilde{u}_{1}^{2} d \sigma & \geq \int_{\Omega^{\star}} g(\theta)_{\star}^{2} u_{1}^{\star 2} d \sigma \\
& \geq \int_{\Omega^{\star}} g(\theta)^{2} u_{1}^{\star 2} d \sigma \geq \int_{B_{\lambda_{1}}} g(\theta)^{2} v_{1}^{2} d \sigma .
\end{aligned}
$$

Here $\tilde{u}_{1}$ represents $u_{1}$ as transplanted to $\tilde{\Omega}$ and $v_{1}$ is the first eigenfunction of $-\Delta$ on $B_{\lambda_{1}}$ with Dirichlet boundary conditions and normalized so that $\int_{\Omega} u_{1}^{2} d \sigma=$ $\int_{B_{\lambda_{1}}} v_{1}^{2} d \sigma$. The functions $g$ and $B$ are likewise based on the eigenfunctions of the ball $B_{\lambda_{1}}$ (so that the $\theta_{1}$ that goes into the boundary value problems (6.3) and (6.4) that define them is the radius of the ball $B_{\lambda_{1}}$ ). In each of (6.8) and (6.9), the equality is trivial, the first inequality follows simply from rearrangement (see Section 5 for our notation), the second inequality is by virtue of the monotonicity properties of $g(\theta)$ and $B(\theta)$, and the last inequality follows from our $\mathbb{S}^{n}$ analog of Chiti's comparison result (see Section 5 for details) and also uses the monotonicity properties of $g$ and $B$ again. Finally, from (6.6), (6.8), and (6.9) we obtain

$$
\lambda_{2}(\Omega)-\lambda_{1}(\Omega) \leq \frac{\int_{B_{\lambda_{1}}} B(\theta) v_{1}^{2} d \sigma}{\int_{B_{\lambda_{1}}} g(\theta)^{2} v_{1}^{2} d \sigma}=\lambda_{2}\left(B_{\lambda_{1}}\right)-\lambda_{1}\left(B_{\lambda_{1}}\right)
$$

Hence $\left(\right.$ since $\left.\lambda_{1}\left(B_{\lambda_{1}}\right)=\lambda_{1}(\Omega)\right)$

$$
\lambda_{2}(\Omega) \leq \lambda_{2}\left(B_{\lambda_{1}}\right)
$$

which concludes the proof of Theorem 1.1 it being clear from any of a number of our previous inequalities that equality obtains in (6.11) if and only if $\Omega$ is itself a ball.

Remarks. (1) Theorems 1.1 (i.e., inequality (6.11) ) and 1.4 also hold under somewhat more general circumstances than for $\Omega$ contained in a hemisphere of $\mathbb{S}^{n}$. In particular, they continue to hold if $\Omega \cap(-\Omega)=\emptyset$, or, more generally, if $\Omega$ satisfies the "excess less than or equal to deficit property" with respect to the center of mass as north (or south!) pole as stated in (2.13) above for all $k \in[0,1]$. We note, however, that even under these conditions $\Omega$ is constrained to have volume 
no larger than that of a hemisphere. See also Remark 4 in Section 2 following the proof of Theorem 2.1

(2) In fact, it is enough that $\Omega$ satisfy $\theta_{1}\left(B_{\lambda_{1}}\right) \leq \pi / 2$ (or, equivalently, $\lambda_{1}(\Omega) \geq$ $n$ ), where $\theta_{1}\left(B_{\lambda_{1}}\right)$ is the geodesic radius of the ball $B_{\lambda_{1}}$, together with the "excess less than or equal to deficit property" (2.13) for all $k \in\left[\cos \theta_{1}\left(B_{\lambda_{1}}\right), 1\right]$. This condition allows us to prove that $\lambda_{2}(\Omega) \leq \lambda_{2}\left(B_{\lambda_{1}}\right)$ even for certain domains which have volume larger than that of a hemisphere (as well as covering all previous cases). A variant of this condition also applies in the case of the Neumann problem (the " $\mu_{1}$ problem") for domains in $\mathbb{S}^{n}$ (see [9] and certain of our remarks in Section 2 above). Then $\theta_{1}\left(\Omega^{\star}\right)$, the geodesic radius of $\Omega^{\star}$, should replace $\theta_{1}\left(B_{\lambda_{1}}\right)$ in the foregoing (and in this case we are still limited by $|\Omega| \leq \frac{1}{2}\left|\mathbb{S}^{n}\right|$ ). The reason for these values of $\theta_{1}$ is that these are the radii we end with in the respective problems, when all is said and done.

\section{ACKNOWLEDGEMENTS}

M.S.A. is grateful for the hospitality of Thomas Hoffmann-Ostenhof and the Erwin Schrödinger Institute (ESI) in Vienna, where some of this work was carried out. We would also like to thank the referee for several useful remarks.

\section{REFERENCES}

1. M. Abramowitz and I. A. Stegun, editors, Handbook of Mathematical Functions, National Bureau of Standards Applied Mathematics Series, vol. 55, U.S. Government Printing Office, Washington, D.C., 1964. MR 34:8607

2. M. A. Armstrong, Basic Topology, Springer-Verlag, New York, 1983. MR 84f:55001

3. M. S. Ashbaugh and R. D. Benguria, Log-concavity of the ground state of Schrödinger operators: a new proof of the Baumgartner-Grosse-Martin inequality, Phys. Lett. A 131 (1988), 273-276. MR 89i:81012

4. M. S. Ashbaugh and R. D. Benguria, Optimal lower bounds for eigenvalue gaps for Schrödinger operators with symmetric single-well potentials and related results, Maximum Principles and Eigenvalue Problems in Partial Differential Equations, P. W. Schaefer, editor, Pitman Research Notes in Mathematics Series, vol. 175, Longman Scientific and Technical, Harlow, Essex, United Kingdom, 1988, pp. 134-145. MR 90c:35157

5. M. S. Ashbaugh and R. D. Benguria, Proof of the Payne-Pólya-Weinberger conjecture, Bull. Amer. Math. Soc. 25 (1991), 19-29. MR 91m:35173

6. M. S. Ashbaugh and R. D. Benguria, A sharp bound for the ratio of the first two eigenvalues of Dirichlet Laplacians and extensions, Annals of Math. 135 (1992), 601-628. MR 93d:35105

7. M. S. Ashbaugh and R. D. Benguria, A second proof of the Payne-Pólya-Weinberger conjecture, Commun. Math. Phys. 147 (1992), 181-190. MR 93k:33002

8. M. S. Ashbaugh and R. D. Benguria, Isoperimetric inequalities for eigenvalue ratios, Partial Differential Equations of Elliptic Type, Cortona, 1992, A. Alvino, E. Fabes, and G. Talenti, editors, Symposia Mathematica, vol. 35, Cambridge University Press, Cambridge, 1994, pp. 1-36. MR 95h:35158

9. M. S. Ashbaugh and R. D. Benguria, Sharp upper bound to the first nonzero Neumann eigenvalue for bounded domains in spaces of constant curvature, J. London Math. Soc. (2) 52 (1995), 402-416. MR 97d:35160

10. M. S. Ashbaugh and R. D. Benguria, On the Payne-Pólya-Weinberger conjecture on the ndimensional sphere, General Inequalities 7 (Oberwolfach, 1995), C. Bandle, W. N. Everitt, L. Losonczi, and W. Walter, editors, International Series of Numerical Mathematics, vol. 123, Birkhäuser, Basel, 1997, pp. 111-128. MR 98k:35139

11. M. S. Ashbaugh and H. A. Levine, Inequalities for the Dirichlet and Neumann eigenvalues of the Laplacian for domains on spheres, Journées "Équations aux Dérivées Partielles" (Saint-Jean-de-Monts, 1997), Exp. No. 1, 15 pp., École Polytechnique, Palaiseau, 1997.

12. F. E. Baginski, Ordering the zeroes of Legendre functions $P_{\nu}^{m}\left(z_{0}\right)$ when considered as a function of $\nu$, J. Math. Anal. Appl. 147 (1990), 296-308. MR 91k:33006 
13. F. E. Baginski, Comparison theorems for the $\nu$-zeroes of Legendre functions $P_{\nu}^{m}\left(z_{0}\right)$ when $-1<z_{0}<1$, Proc. Amer. Math. Soc. 111 (1991), 395-402. MR 91i:33003

14. C. Bandle, Isoperimetric Inequalities and Applications, Pitman Monographs and Studies in Mathematics, vol. 7, Pitman, Boston, 1980. MR 81e:35095

15. C. Bandle and M. Flucher, Table of inequalities in elliptic boundary value problems, Recent Progress in Inequalities (Niš, 1996), G. V. Milovanovic, editor, Mathematics and its Applications, vol. 430, Kluwer Academic Publishers, Dordrecht, The Netherlands, 1998, pp. 97-125. MR 99h:35028

16. B. Baumgartner, Level comparison theorems, Annals of Physics 168 (1986), 484-526. MR 87j:81046

17. B. Baumgartner, Relative concavity of ground state energies as functions of a coupling constant, Phys. Lett. A 170 (1992), 1-4. MR 93i:81022

18. B. Baumgartner, H. Grosse, and A. Martin, The Laplacian of the potential and the order of energy levels, Phys. Lett. B 146 (1984), 363-366. MR 85k:81158

19. B. Baumgartner, H. Grosse, and A. Martin, Order of levels in potential models, Nucl. Phys. B 254 (1985), 528-542. MR 87a:81024

20. G. Birkhoff and G.-C. Rota, Ordinary Differential Equations, fourth edition, Wiley, New York, 1989. MR 90h:34001

21. Yu. D. Burago and V. A. Zalgaller, Geometric Inequalities, Grundlehren der mathematischen Wissenschaften 285, Springer-Verlag, Berlin, 1988. MR 89b:52020

22. I. Chavel, Lowest-eigenvalue inequalities, Proc. Symp. Pure Math., vol. 36, Geometry of the Laplace Operator, R. Osserman and A. Weinstein, editors, Amer. Math. Soc., Providence, Rhode Island, 1980, pp. 79-89. MR 81f:58039

23. I. Chavel, Eigenvalues in Riemannian Geometry, Academic Press, New York, 1984. MR 86g:58140

24. G. Chiti, Orlicz norms of the solutions of a class of elliptic equations, Boll. Un. Mat. Ital. (5) 16-A (1979), 178-185. MR 80h:35004

25. G. Chiti, A reverse Hölder inequality for the eigenfunctions of linear second order elliptic operators, J. Appl. Math. and Phys. (ZAMP) 33 (1982), 143-148. MR 83i:35141

26. G. Chiti, An isoperimetric inequality for the eigenfunctions of linear second order elliptic operators, Boll. Un. Mat. Ital. (6) 1-A (1982), 145-151. MR 83i:35140

27. G. Chiti, A bound for the ratio of the first two eigenvalues of a membrane, SIAM J. Math. Anal. 14 (1983), 1163-1167. MR 85f:35158

28. R. Courant and D. Hilbert, Methods of Mathematical Physics, vol. I, Interscience Publishers, New York, 1953. MR 16:426a

29. J. Dugundji, Topology, Allyn and Bacon, Boston, 1966. MR 33:1824

30. G. Faber, Beweis, dass unter allen homogenen Membranen von gleicher Fläche und gleicher Spannung die kreisförmige den tiefsten Grundton gibt, Sitzungsberichte der mathematischphysikalischen Klasse der Bayerischen Akademie der Wissenschaften zu München Jahrgang, 1923, pp. 169-172.

31. S. Friedland and W. K. Hayman, Eigenvalue inequalities for the Dirichlet problem on spheres and the growth of subharmonic functions, Comment. Math. Helvetici 51 (1976), 133-161. MR 54:568

32. G. H. Hardy, J. E. Littlewood, and G. Pólya, Inequalities, second edition, Cambridge University Press, Cambridge, 1952. MR 13:727a

33. E. M. Harrell II and P. L. Michel, Commutator bounds for eigenvalues, with applications to spectral geometry, Commun. Partial Diff. Eqs. 19 (1994), 2037-2055. MR 95i:58182

34. P. D. Hislop and I. M. Sigal, Introduction to Spectral Theory, With Applications to Schrödinger Operators, Applied Mathematical Sciences, vol. 113, Springer-Verlag, New York, 1996. MR 98h:47003

35. J. G. Hocking and G. S. Young, Topology, Addison-Wesley, Reading, Massachusetts, 1961. MR 23:A2857

36. T. Kato, Perturbation Theory for Linear Operators, 2nd edition, Grundlehren der mathematischen Wissenschaften 132, Springer-Verlag, Berlin, 1976. MR 53:11389

37. E. Krahn, Über eine von Rayleigh formulierte Minimaleigenschaft des Kreises, Math. Ann. 94 (1925), 97-100.

38. E. Krahn, Über Minimaleigenschaften der Kugel in drei und mehr Dimensionen, Acta Comm. Univ. Tartu (Dorpat) A9 (1926), 1-44. [English translation: Minimal properties of the sphere 
in three and more dimensions, Edgar Krahn 1894-1961: A Centenary Volume, Ü. Lumiste and J. Peetre, editors, IOS Press, Amsterdam, The Netherlands, 1994, pp. 139-174.]

39. J. R. Munkres, Topology, A First Course, Prentice-Hall, Englewood Cliffs, New Jersey, 1975. MR 57:4063

40. J. R. Munkres, Elements of Algebraic Topology, Addison-Wesley, Menlo Park, California, 1984. MR 85m:55001

41. R. Osserman, The isoperimetric inequality, Bull. Amer. Math. Soc. 84 (1978), 1182-1238. MR 58:18161

42. L. E. Payne, G. Pólya, and H. F. Weinberger, Sur le quotient de deux fréquences propres consécutives, Comptes Rendus Acad. Sci. Paris 241 (1955), 917-919. MR 17:372d

43. L. E. Payne, G. Pólya, and H. F. Weinberger, On the ratio of consecutive eigenvalues, J. Math. and Phys. 35 (1956), 289-298. MR 18:905c

44. J. W. S. Rayleigh, The Theory of Sound, second edition revised and enlarged (in two volumes), Dover Publications, New York, 1945 (republication of the 1894/1896 edition). MR 7:500e

45. M. Reed and B. Simon, Methods of Modern Mathematical Physics, vol. IV: Analysis of Operators, Academic Press, New York, 1978. MR 58:12429c

46. R. D. Richtmyer, Principles of Advanced Mathematical Physics, vol. II, Springer-Verlag, New York, 1981. MR 84h:00024b

47. K. T. Smith, Primer of Modern Analysis, Bogden and Quigley, Tarrytown-on-Hudson, New York, 1971.

48. E. H. Spanier, Algebraic Topology, McGraw-Hill, New York, 1966. MR 35:1007

49. E. Sperner, Zur Symmetrisierung von Funktionen auf Sphären, Math. Z. 134 (1973), 317327. MR 49:5310

50. G. Szegő, Inequalities for certain eigenvalues of a membrane of given area, J. Rational Mech. Anal. 3 (1954), 343-356. MR 15:877c

51. G. Talenti, Elliptic equations and rearrangements, Ann. Scuola Norm. Sup. Pisa (4) 3 (1976), 697-718. MR 58:29170

52. H. F. Weinberger, An isoperimetric inequality for the $n$-dimensional free membrane problem, J. Rational Mech. Anal. 5 (1956), 633-636. MR 18:63c

53. E. F. Whittlesey, Fixed points and antipodal points, Amer. Math. Monthly 70 (1963), 807-821. MR 28:4532

Department of Mathematics, University of Missouri, Columbia, Missouri 65211-0001 E-mail address: mark@math.missouri.edu

Departamento de Física, P. Universidad Católica de Chile, Casilla 306, Santiago 22, Chile

E-mail address: rbenguri@fis.puc.cl 